\title{
Exploration-Dependent Modulation of Evoked Responses in Fascia Dentata: Dissociation of Motor, EEG, and Sensory Factors and Evidence for a Synaptic Efficacy Change
}

\author{
E. J. Green, ${ }^{a}$ B. L. McNaughton, and C. A. Barnes \\ Department of Psychology, University of Colorado, Boulder, Colorado 80309
}

Sharp et al. (1986, 1989) described several alterations in hippocampal responses to perforant path stimulation which accompany exploratory activity. Animals transferred from one environment to another exhibited substantial increases in population EPSPS, and large decreases in both the amplitude and the peak latency of population spikes. These changes are unlike the "gating" effects of behavioral state reported by Winson and Abzug $(1977,1978)$ in that they considerably outlast the behaviors that produce them and (as shown here) can be dissociated from the EEG state of the hippocampus. In this report we describe several new observations that aid in the interpretation of the possible mechanisms and functional significance of these effects.

Transportation of animals from their home environment to a different location was accompanied by: (1) an increase in the incidence of exploratory behavior, and EEG theta rhythm, (2) a substantial, reliable, and persistent (more than $15 \mathrm{~min}$ ) elevation in the size of the evoked field EPSP recorded at the site of synaptic activation in the stratum moleculare and in the stratum granulosum/hilus, and (3) a reduction in the evoked population spike, and (paradoxically) in the latencies to spike onset and peak. There were no associated changes in the spike threshold (defined as the magnitude of the field EPSP at spike onset), the amplitude of the presynaptic fiber volley, or the fractional change in the second synaptic response during paired-pulse stimulation (suggesting the absence of a change in fractional transmitter release). Neither the motor component of exploratory behaviors nor the hippocampal theta rhythm itself was sufficient to account for the changes in the synaptic and spike components of the response. Thus, the changes may depend on reorientation of the animal's sensorium during exploration, rather than on movement per se or the associated hippocampal theta rhythm. The results of these experiments suggest that exploration-related EPSP growth is due neither to granule cell hyperpolarization nor to changes in the distribution of current sources and sinks on granule cells. Rather, the data suggest that exploratory behavior is accompanied by a substantial

\footnotetext{
Received April 4, 1989; revised Nov. 30, 1989; accepted Dec. 1, 1989

This work was supported by BNS-8617464 to B.L.M. and AG-03376 to C.A.B. E.J.G. was supported by NRSA postdoctoral fellowship F32 AG05345. We thank C. A. Erickson for assistance with experiment 1, and P. E. Sharp for helpful comments during manuscript preparation.

Correspondence should be addressed to Dr. E. I. Green, Department of Psychology. University of Miami, Box 248185. Coral Gables. FL 33124

Present address: Iepartment of Psycholngy. Iniversity of Miami. Box 248185. Coral Gables, FL 33124.

Copyright c 1990 Society for Neuroscience $0270-6474 / 90 / 051455-17 \$ 02.00 / 0$
}

increase in evoked synaptic efficacy in granule cells, an increase that long outlasts the behavior that produces it. A possible role for these alterations in information processing and memory is discussed.

Exploratory behavior in rats is accompanied by several slowly decaying alterations in the components of electrically evoked synaptic responses in the fascia dentata of the hippocampal formation (Sharp et al., 1985, 1986, 1989). These alterations include a robust increase in the magnitude of population EPSPs and a decrease in both the amplitude and the peak latency of evoked population spikes. These changes cannot be attributed to handling, nor are they a consequence of the repeated electrical stimuli delivered to the perforant path. Moreover. the synaptic response alterations outlast changes in behavior, distinguishing them from the behavioral state "gating" effect described by Winson and Abzug (1977, 1978). Sharp and colleagues further reported that these changes were not related to the instantaneous behavior of the animal. Rather, a strong correlative relationship was found between the recent history of exploratory activity [Vanderwolf's (1969) Type I, "theta" behaviors, including walking, sniffing, rearing] and the synaptic (EPSP) changes observed in different environmental situations. The synaptic response appeared to behave like a mathematical integrator of recent exploratory behavior, with a 5-15-min time constant. Most of the variance in the Sharp et al. (1989) synaptic response data was accounted for by numerical integration of the relation

$$
d(\mathrm{EPSP}) / d t=-k_{1}(\mathrm{EPSP}-1)+k_{2}(k \mathrm{X})
$$

where EPSP reflects the synaptic response normalized to its lowest baseline value, $E x$ is a measure of exploratory behavior, $k_{1}$ and $k_{2}$ are positive constants. The strength of this relationship and the wealth of experimental evidence for a hippocampal role in spatial learning and memory processes (O'Keefe and Nadel. 1978; Olton et al., 1978; Morris et al., 1982; Jarrard, 1983; Sutherland et al., 1983; Sharp et al., 1985: McNaughton et al., 1986) led to the suggestion that the EPSP changes might reflect the temporary storage of information acquired as an animal actively reorients its sensorium in relation to its environment.

While the foregoing results indicate that the animal's interaction with its environment can lead to consistent and robust physiological changes within the fascia dentata, both the mechanism(s) of these changes and their role in hippocampal function remain to be determined. One problem, crucial to an overall interpretation of these changes, is the question of whether they are associated with informational aspects of environmental sensory input, or whether they are merely a consequence either of 
locumotion or of the hippocampal state change (theta rhythm) which invariably accompanies locomotion and sensory scanning (Vanderwolf, 1969; Winson, 1974). By dissociating the specific contributions of motor behavior, EEG state, and the informational properties of environmental sensory input, it may be possible to determine the extent to which the physiological changes reflect the processing of information about the environment by the hippocampus and its associated circuitry.

In this report, we present experiments that address this problem. First, we describe results suggesting that growth in the synaptic response is causally related neither to the motor component of exploratory behaviors nor to the hippocampal theta rhythm itself. These data support the possibility that the synaptic changes may be related to the processing of environmental information. We further describe a series of experiments which indicate that a component of the evoked population spike alteration is modulated by the animals' familiarity with the current environmental situation. Finally, we provide additional evidence that at least part of the exploratory activity-associated EPSP growth reflects a genuine increase in perforant path synaptic efficacy, which is unaccompanied by an increase in fractional transmitter release. In conjunction with similar data (McNaughton, 1982) on artificially induced long-term synaptic enhancement (LTE/LTP), these findings raise the possibility that the 2 effects may share elements of a common mechanism.

\section{Materials and Methods}

Subjects. Twenty-three male, Fischer-344 rats (10-14 months of age) were used in these experiments. Rats were singly housed in clear plastic tubs $(43 \times 22 \times 10 \mathrm{~cm})$ and allowed free access to food and water, except where noted below. The colony room was maintained on a 12 / 12 -hr light/dark cycle, and all testing was carried out during the light phase of the cycle.

Surgery: Chronic surgical implantation of electrodes for stimulation of perforant path (PP) and recording of the resulting field potentials in fascia dentata $(\Gamma D)$ was conducted under sodium pentobarbital anesthesia $(33 \mathrm{mg} / \mathrm{kg}$ ). Extracellular recording electrodes were made from $114 \mu \mathrm{m}$ (outer diameter) stainless steel wire insulated with Teflon and cut evenly at the tip. Monopolar stimulating electrodes for the perforant path were made of the same wire, except that $200-400 \mu \mathrm{m}$ of insulation was removed from the tip. In experiments involving simultaneous recordings from stratum granulosum/hilus and stratum moleculare, the electrodes were made of $62 \mu \mathrm{m}$ Teflon-insulated tungsten wire. Nominal stereotaxic coordinates (flat skull) for the electrode placements, with reference to bregma and the midline, were (in $\mathrm{mm}$ ): PP, 8.1 posterior, 4.4 lateral; FD, 4.0 posterior, 2.2 lateral. The depth of $F D$ recording and PP stimulating electrodes was adjusted using physiological criteria. Stainless steel screws overlying neocortex served as ground and indifferent electrodes, and as return paths for stimulus current. Gold amphenol connectors were used to connect the stimulating and recording electrode wires to a plastic receptacle, and the entire assembly was cemented to the skull with dental acrylic. Wound edges were infiltrated with Furacin antibacterial cream, and bicillin was administered intramuscularly to each hindlimb. In one rat a 25 -gauge guide cannula was implanted with its tip located $0.5 \mathrm{~mm}$ above the medial septal nucleus. Subsequently, experiments were carried out in which $0.7 \mu \mathrm{l}$ of the local anesthetic tetracaine ( $2 \%$ in physiological saline) was injected into the medial septum (using the procedures described by Mizumori et al., 1989a) in order to abolish the hippocampal theta rhythm.

Stimulation and recording. Diphasic stimuli (100 $\mu \mathrm{sec} / \mathrm{half}-\mathrm{cycle})$ were delivered at $0.1 \mathrm{~Hz}$ (except where otherwise noted). Stimulus intensity was individually determined for each rat and adjusted to elicit population spikes that were approximately half-maximal at the beginning of the first day's recording session. Evoked field potentials wcre filtercd (half-amplitude attenuation) at $1 \mathrm{~Hz}$ (high pass) and $3 \mathrm{kHz}$ (low pass), amplified, and sampled by computer at either 10 or $20 \mathrm{kHz}$. EEG epochs of $2.56 \mathrm{sec}$ were collected immediately prior to each evoked field potential, band-pass filtered between 0.1 and $30 \mathrm{~Hz}$, amplified, and sam- pled by computer at $100 \mathrm{~Hz}$. An FET follower headstage mounted on top of the head plug assembly was used to minimize electrical noise and movement artifact. Evoked potentials and EEG data were stored on magnetic disk and analyzed off-line.

Representative response waveforms and hippocampal EEG under various experimental conditions are illustrated in Figure 1. For responses recorded from the stratum granulosum/hilus, population EPSP magnitude was assessed by measuring the amplitude difference between 2 points (indicated by dashed lines) at fixed latencies from the stimulus artifact, on the rising phase of the waveform (Fig. 1 $\mathrm{A}$ ). The amount of granule cell discharge was estimated by computing the population spike area, taken as the area between the data curve and a line tangent to the curve at 2 points. Spike area is highly correlated with spike height under normal conditions ( $r>0.90$; Barnes, 1979), but, unlike spike height, is much less influenced by discharge synchrony. The unique tangent points noted above were defined as the spike onset and offset times. Spike onset and peak latencies were determined by computing the time between the apparent EPSP onset and the spike onset, and peak spike amplitude times, respectively. EPSP amplitude at spike onset (spike threshold) was computed as the voltage difference between a prestimulus baseline and the EPSP amplitude at the time of spike onset for each waveform. EPSP responses recorded from the stratum moleculare were assessed by measuring the amplitude difference between 2 points at fixed latencies from the stimulus artifact. To prevent contamination with synaptic current flow, presynaptic afferent fiber volley magnitude was taken as the peak positive amplitude (Fig. 1B, arrow).

Artifact-free EEG epochs were subjected to fast-Fourier analysis, and 1 -min averages of the spectral power between 6.6 and $7.4 \mathrm{~Hz}$ were computcd (cxcept wherc otherwise noted). Samples of EEG collected during sessions of walking on the treadmill and sitting quietly in the same apparatus along with the power spectra computed from those epochs are shown in Figure $1, C$ and $D$, respectively.

Amplitude, area, and latency measures from each animal were expressed relative to a baseline condition which was taken as the intercept of a linear regression of the first 10 data points collected each day (except where noted in Figs. 2, 3, and 7). Changes in the magnitude of the various evoked response measures were thus expressed relative to an initial value of 1.0. For example, a value of 1.5 does not indicate a 1.5 $\mathrm{mV}$ response, but, rather, indicates a response that was $50 \%$ greater than the initial value for that recording session.

Environments. During the course of these experiments, evoked responses were collected from chronically prepared animals confined to cither (1) a motorized treadmill, (2) a plastic tub, which scrved as the home environment in the animal colony, (3) an $18 \mathrm{~cm}$ diameter Plexiglas cylinder, or (4) an elevated triangular platform $(59 \mathrm{~cm}$ on each side). The treadmill consisted of an $8 \mathrm{~cm}$ wide, motor-driven belt, upon which was mounted a rectangular box $(34 \times 9 \times 20 \mathrm{~cm})$ to contain the rat. One wall of the box was constructed of clear glass, covered with window screen. The Plexiglas cylinder (recording chamber, RC) was located inside an electrically screened enclosure.

For statistical analysis, repeated measure ANOVAs were used to assess the effects of days and/or conditions on response values. Post hoc comparisons between groups were then accomplished using Scheffé tests. Paired $t$-tests were used for statistical evaluation of alterations in response magnitude at 2 particular time points in a recording session (e.g. EPSP amplitude at minutes 1 and 10 following an environmental manipulation)

Behavioral observations. Behavioral observations were taken at the time of each stimulus delivery (i.e., every $10 \mathrm{sec}$ ). Behaviors were divided into 2 categories: exploratory behaviors (Vanderwolf's Type I), which are defined as behaviors such as walking, sniffing, and rearing. and which, in rats, are associated with theta rhythm in the hippocampal EEG; and nonexploratory behaviors (Vanderwolfs Type II), which, in the present context, included inactivity, during which the animal is motionless but awake, and grooming.

\section{Results}

Figure 2 illustrates the major effects of exploratory activity on the evoked response parameters evaluated in this report. An individual rat was rapidly transported in its home cage from the animal colony to the recording room and immediately connected to the recording apparatus. In the first $10-15 \mathrm{~min}$ following the transfer, the animal exhibited substantial exploration 
A
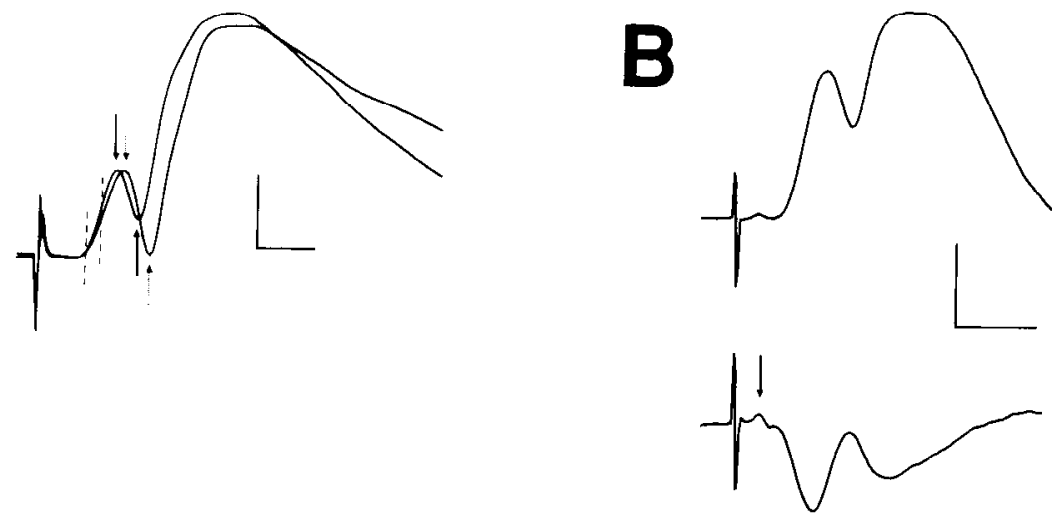

C

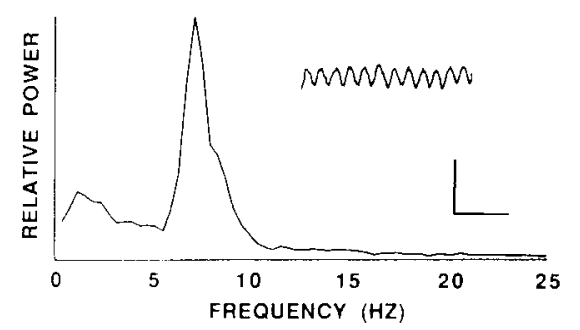

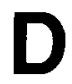

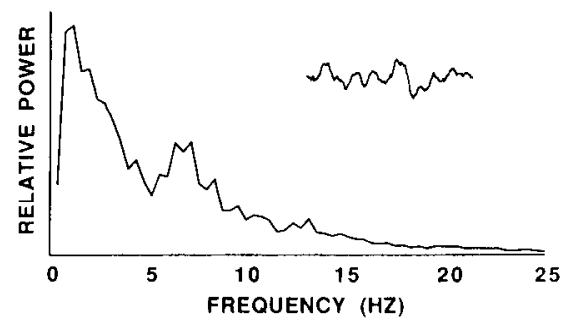

Figure 1. Representative cvoked responses. A, Evoked responses collected from the stratum granulosumhilus 1 min (segmented arrows) and $16 \mathrm{~min}$ (solid arrows) following the transfer of an animal from its home environment. The falling arrows depict the onset of the population spike, and the rising arrows indicate the peak of the spike. The dashed lines show the approximale points of measurement of the EPSP. The fractional change in EPSP amplitude over this time period was 1.34 (1.e.. 34\% growth in EPSP value). B, Evoked responses collected simultaneously from clectrodes in stratum gramulosum (top) and stratum moleculare (bottom). The solid arrow indicates the positive peak of the compound action potential (fiber potential) generated by the presynaptic axons activated by the stimulus. $C, D$. Representative power spectra computed from samples of EEG collected during sessions of walking on the treadmill $(C)$ and sitting quietly in the same apparatus $(D)$. Insets show the EEG epochs from which those spectra were computed. Calibration: $4.7 \mathrm{mV}, 3$ msec: $B, 5 \mathrm{mV} .3 \mathrm{msec}$ positive up $C$. $D: 1 \mathrm{mV} .0 .5 \mathrm{sec}$. and hippocampal theta activity (Fig. 2A). This was accompanied by a large $(>50 \%)$ gradual increase in EPSP amplitude (Fig. $2 B$ ) and decreases in both spike onset latency (Fig. 2() and spike area (Fig. 2D). Notice that the EEG theta activity is initially high and declines approximately exponentially, whereas the EPSP exhibits a gradual growth and a slow decline which long outlasts the presence of intense theta activity. This describes the behavior of an integrator with a slow time constant. Over the next 60 min, the animal was awake, but relatively quiescent, and EPSP and spike onset response amplitudes recovered toward their initial values. During minutes 100-120 (depicted by the bar) the room lights were extinguished, resulting in an increase in exploratory behavior and theta activity in the EEG. The abrupt increase in exploration led to a renewed gradual increase in EPSP amplitude and additional decreases in spike onset latency and spike area. Again, the alterations in the evoked response measures outlasted the behavioral and EEG changes by many minutes.

Further details concerning the individual experiments are described with the corresponding results.

\section{Experiment l: control for sensory adaptation and transient response depression following environmental transfer}

The following experiment was conducted in 4 rats in order to control for possible effects of sensory adaptation and to demonstrate that the EPSP change represents a true growth above baseline, rather than a recovery from some depressive effect of the environmental transfer. At 5 P.M. on the evening prior to the recording session, each rat was brought into the recording room in its home cage, which was placed on top of the triangular platform. The rat was connected to the recording apparatus and left with the home cage uncovered overnight in the recording room. At 9 A.M. the following morning the experimenter entered the recording room, tapped gently on the side of the home cage to ensure that the rat was not asleep, and commenced a 3-hr recording session. Behavioral state was monitored and recorded after each stimulus delivery (once each $10 \mathrm{sec}$ ). After $30 \mathrm{~min}$, the rat was taken out of its home cage and placed onto the triangular platform on which a variety of "junk" objects had been placed. The rat was allowed to explore the platform for 33 min, after which it was replaced into the home cage under the baseline conditions for the remainder of the recording session.

The results from one rat are illustrated in Figure 3. During the $30-\mathrm{min}$ baseline condition the animals remained stationary, but awake, in the home cage, occasionally looking around or grooming. Exploratory activity accounted for less than $10 \%$ of behavior during the baseline period. During the $33 \mathrm{~min}$ on the triangular platform, the rats exhibited extensive exploratory activity and occasional bouts of grooming. Exploratory activity accounted for over $75 \%$ of the behavioral scores. Return to the home cage was accompanied by a bout of exploratory activity. followed by immobility and grooming.

The amplitude of the EPSP remained stable during the baseline period, increased gradually during the exploration session to almost twice the bascline valuc, and decaycd gradually back to baseline following return to the home cage. The spike area exhibited a slight reduction during the first few minutes of the baseline period and fell dramatically during the exploratory session. Unlike the EPSP, the spike area did not completely return to its baseline value during the $2 \mathrm{hr}$ in the home cage following the exploratory session, although some recovery was observed. The spike onsel latency exhibited changes that were roughly inverse to the EPSP changes. 

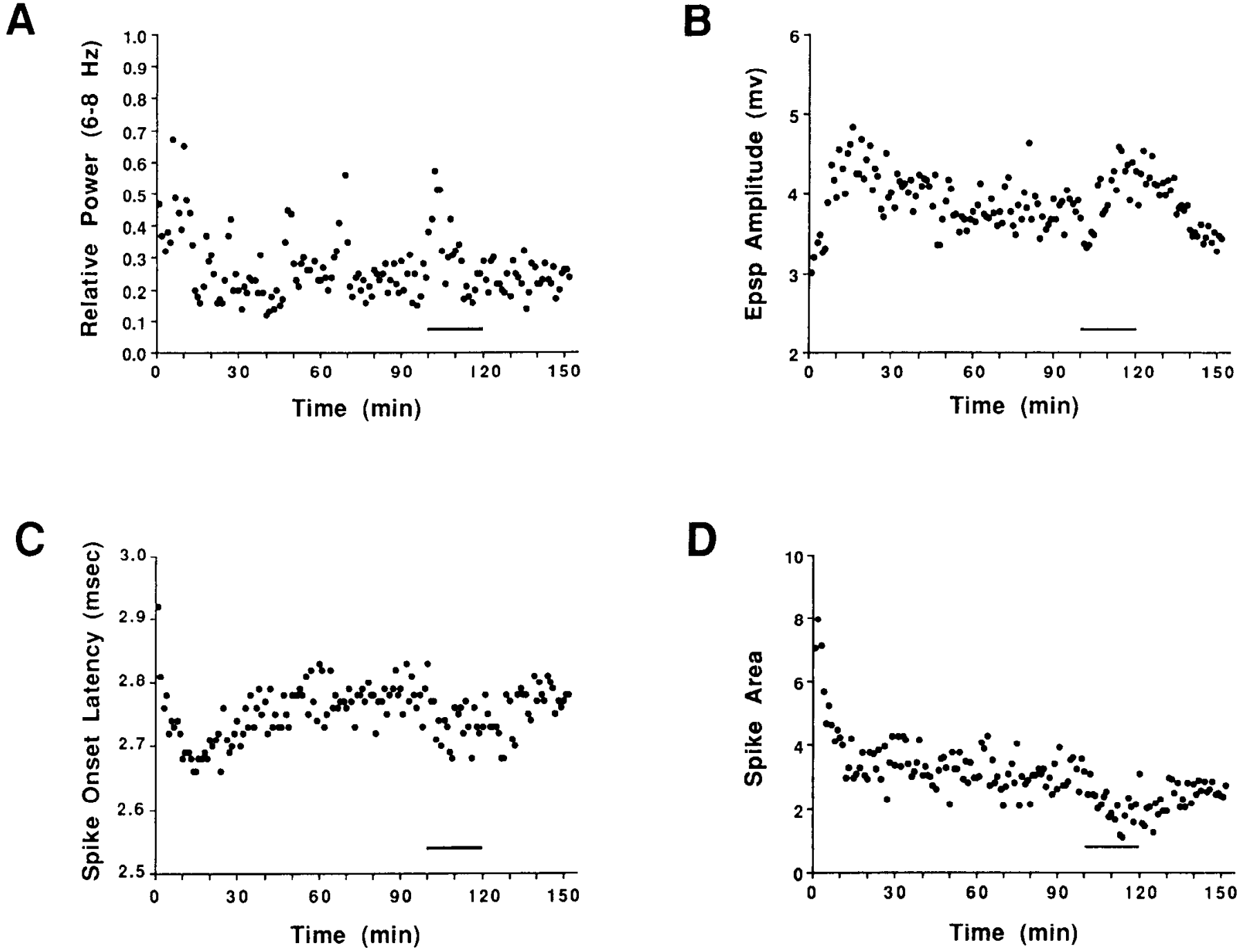

D

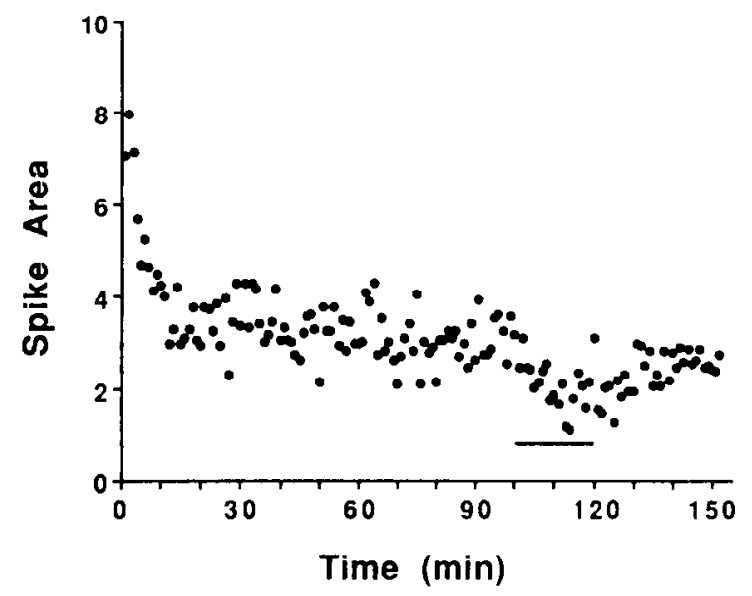

Figure 2. Some of the major changes in hippocampal evoked responses that are associated with, but outlast, exploration. Raw (nonnormalized) data from an individual animal are averaged in 1 -min bins and plotted as a function of time. Following transfer of an animal from the colony to the recording room $(t=0)$, there was initially a substantial amount of exploration and hippocampal theta activity $(A)$ (which is highly correlated with such behavior). Exploration and the associated theta power declined over the first $20 \mathrm{~min}$. During this time there was a gradual but large increase in EPSP amplitude $(B)$ and a corresponding decrease in spike onset latency $(C)$. Paradoxically, spike size declined during this period $(D)$. EPSP and spike onset values recovered gradually over the next $60 \mathrm{~min}$ while the animal was awake, but relatively quiescent. During minutes $100-$ 120 (depicted by the bar) the room lights were extinguished. This resulted in an increase in exploratory behavior and theta activity in the EEG. The increase in exploration led to an additional gradual increase in EPSP amplitude and decreases in spike onset latency and spike area. Again, these changes outlasted the behavioral and EEG state changes. As shown by Sharp et al. (1989), the EPSP change appears to be related to the time integral of the exploratory behavior, with a decay time constant of many minutes.

\section{Experiment 2: role of motor activity in evoked response alterations}

In order to dissociate the effects on evoked responses of the motor components of exploratory activity from those due to variation in sensory input, we examined chronically prepared animals in several environmental situations. This experiment was designed to separate out the effects of locomotion by holding the sensory experience relatively constant.

In experiment $2 \mathrm{a}, 5$ rats were surgically prepared as described above. After recovery from surgery the rats were given an extensive period of training in the treadmill apparatus. The formal experiments began 3 weeks after initiation of training. For the recording sessions, each rat was carried in its plastic tub to the recording room, connected to the recording apparatus, and re- turned to the tub. For logistical reasons, it was not possible to conduct overnight baseline recordings as described in experiment 1 . However, in this experiment, the elapsed time between initial handling of the tub in the colony room until commencement of recording ranged between 60 and $120 \mathrm{sec}$. Thus, there would have been little opportunity for response change prior to the initiation of recording. Following the initial $10 \mathrm{~min}$ in the plastic tub (TUB), rats received $10 \mathrm{~min}$ in the stationary treadmill (TM) and then $15 \mathrm{~min}$ in either the Plexiglas recording chamber (condition A) or the moving treadmill (condition B). The animals were then returned to the stationary treadmill for $10 \mathrm{~min}$. The environments were situated in the same room (within an area $5 \mathrm{ft}^{2}$ ), and transfer between environments was accomplished in less than $10 \mathrm{sec}$; hence, there was no interruption in data collection during the transfers. The animals' be- 
havior at the time of each perforant path stimulus was categorized as either exploratory or nonexploratory, as defined above. These experiments were conducted for 6 successive days, with each type of session (A or B) conducted on alternate days.

Behavior and evoked EPSP. Data for experimental conditions RC and TM are illustrated in the corresponding columns of Figure 4. The first and second rows show the behavioral and EPSP data averaged across days and animals. Substantial exploratory behavior was exhibited over the first $10 \mathrm{~min}$ of all sessions (TUB). Increases in the incidence of exploratory behavior also generally occurred immediately following each environmental transition. The incidence of exploration declined fairly rapidly over the next several minutes. There was no consistent change in this pattern over days.

The initial transfer from the colony room resulted in a substantial increase in EPSP amplitude. After the first $20 \mathrm{~min}$, EPSP values increased on average to a value of $1.39 \pm 0.028$ (SEM) in condition $\mathrm{A}$ and $1.54 \pm 0.033$ in condition $\mathrm{B}$. Comparisons of EPSP values for minutes 1 and 20 indicated that this increase was significant in each condition $(p<0.01)$. At the moment of each successive transfer (except the last), there was a transient reduction in EPSP amplitude. This transient reduction recovered rapidly. Activation of the treadmill in condition $B$ resulted in a precipitous reduction in EPSP amplitude. When the treadmill was turned off, there was an abrupt recovery to a point approximately equivalent to the value at the end of the TM phase. In other words, intense locomotor activity in the treadmill produced no additional EPSP growth. Transfer to the RC resulted in a similar, although less substantial, attenuation in EPSP amplitude. However, the latter attenuation was not maintained as in the corresponding period in the TM condition. In addition, note that the elevation of "theta behavior" was correspondingly not maintained throughout this phase.

There was no significant change across the $6 \mathrm{~d}$ of the experiment in the initial EPSP amplitude $(F(5,25)=1.59, p>0.2)$, indicating that any changes that occurred during the experiments had dissipated by the next day.

The foregoing results suggested that there are 2 components to the changes in the EPSP in relation to behavior: a cumulative growth, related to the recent history of exploratory activity, and a superimposed, transient attenuation, related to the instantaneous state of locomotion.

It was of theoretical interest to determine whether the transient locomotion-related EPSP attenuation was proportional to the absolute magnitude of the EPSP at the time of the effect, or to the net EPSP growth over baseline. One-minute averages of EPSP amplitude were computed for each animal for each of the $3 \mathrm{~d}$ that the animals were exposed to the moving treadmill. The magnitude of momentary EPSP reduction was then plotted as a function of the EPSP amplitude just prior to treadmill activation, and the data were fitted using a linear regression model. The intercept of the regression line with the EPSP axis was very close to 1.0 rather than to zero. indicating that the EPSP reduction was proportional to the net EPSP growth just prior to walking $(F(1,26)=33.749, p=0.0001$; see Fig. 5.A) rather than to the absolute value of the EPSP itself. These results prompted us to perform identical comparisons for the TM-to-RC transi-

Figure 3. Experiment 1: Exploration-associated changes in perforant path evoked responses are not dependent on general changes in sensory
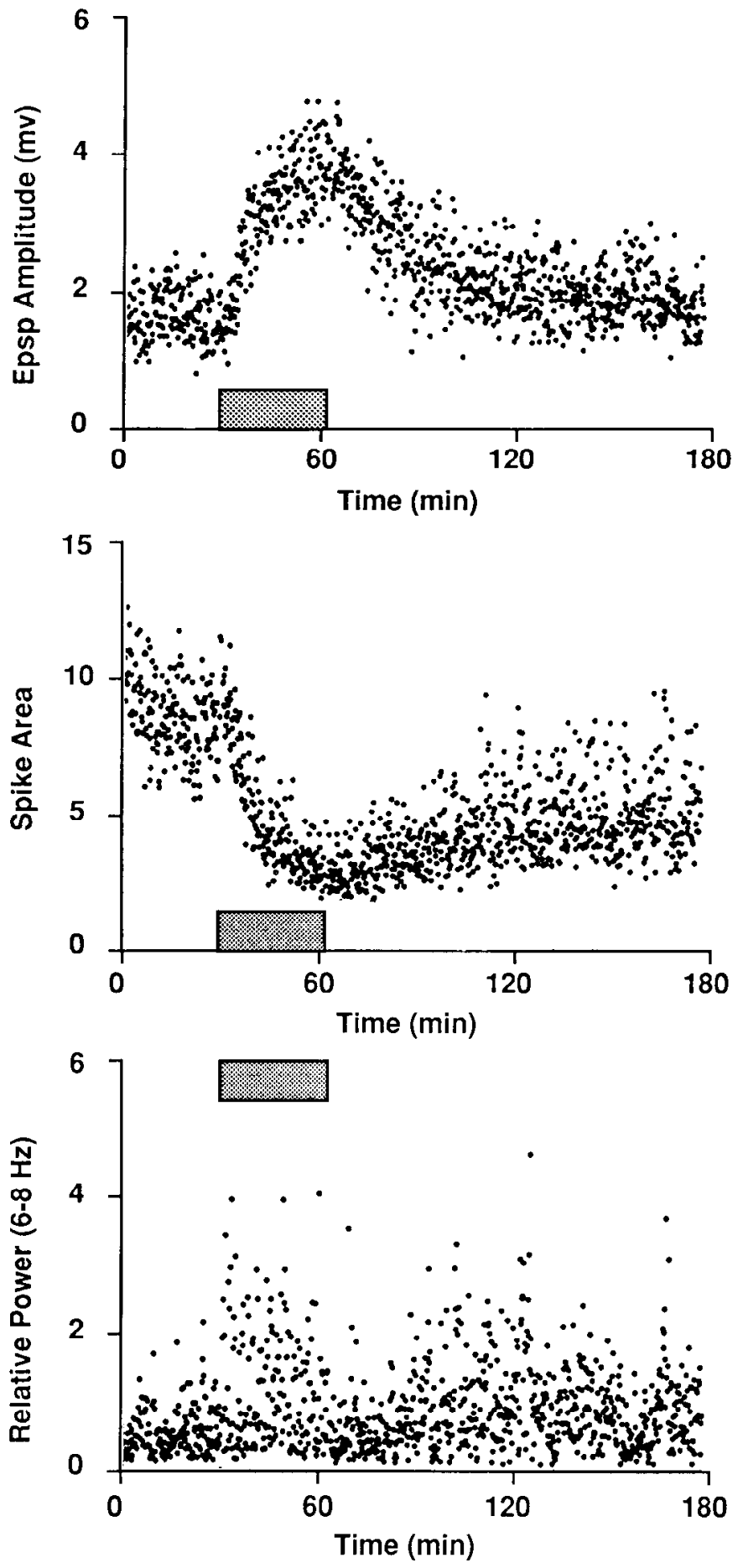

adaptation. Nor are these effects due to recovery from transient alterations that might occur during the course of moving animals from their normal colony room to the recording room. The raw data shown here are from one of the rats that were placed in the recording apparatus in their home cages overnight prior to the actual recording session. At the start of the session, the cage was gently tapped to ensure that the animal was awake. During the first $30 \mathrm{~min}$, the animals sat quietly or groomed at least $90 \%$ of the time. During the next $33 \mathrm{~min}$, they were simply picked up and placed on the platform on which the home cage had been resting and allowed to explore various "junk" objects placed there. The period of exploration is indicated by the shaded bar. This exploration was accompanied by gradual, but substantial, changes in the response parameters. The induced changes returned toward baseline over the subsequent $2 \mathrm{hr}$, during which the rats sat in the home cage in the original location. 


\section{A}
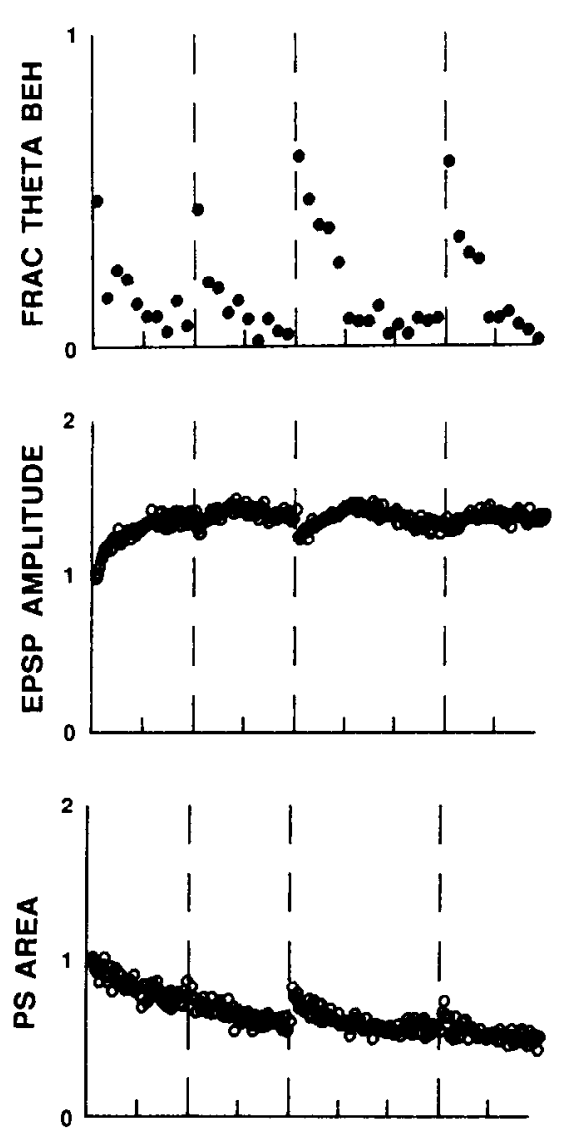

Figure 4. Experiment 2A: Average group means for the behavioral and evoked response parameters for the $\mathrm{RC}$ $(A)$ and TM $(B)$ experimental conditions (see text) plotted as functions of time ( $5 \mathrm{~min} /$ division). The top 2 rows show, respectively, group means of percent locomotion (FRAC THETA BEH) averaged over 1 -min bins, and evoked EPSP amplitudes (plotted relative to a baseline value of 1.0 ). The third through the fifth rows show, respectively, group means for population spike size ( $P S$ $A R E A$ ), spike onset latency, and spike peak latency. Each plot represents data from one hemisphere from each of 5 animals recorded over $3 \mathrm{~d}$. The arrows denote points at which the animals were transferred between environments. The mean peak growth of the EPSP during this experiment was approximately $50 \% . P S=$ population spike; $T U B=$ home cage; $T M=$ treadmill; $R C=$ recording chamber.
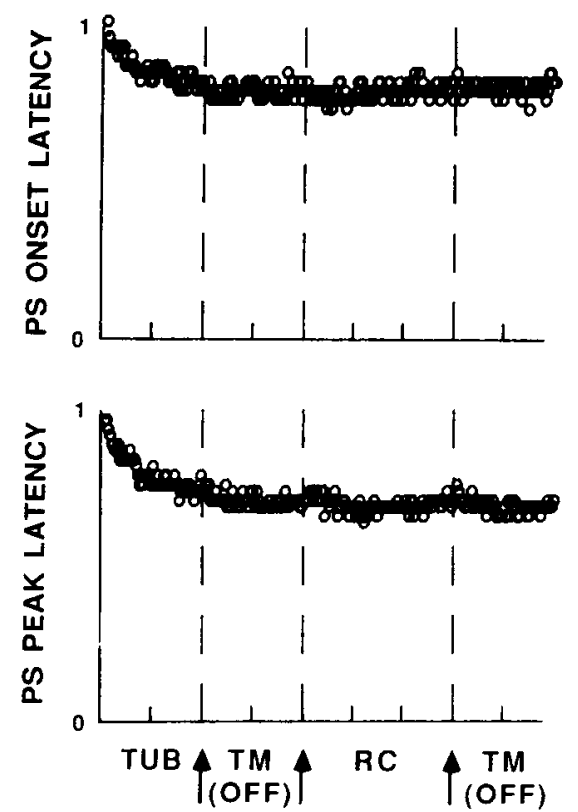
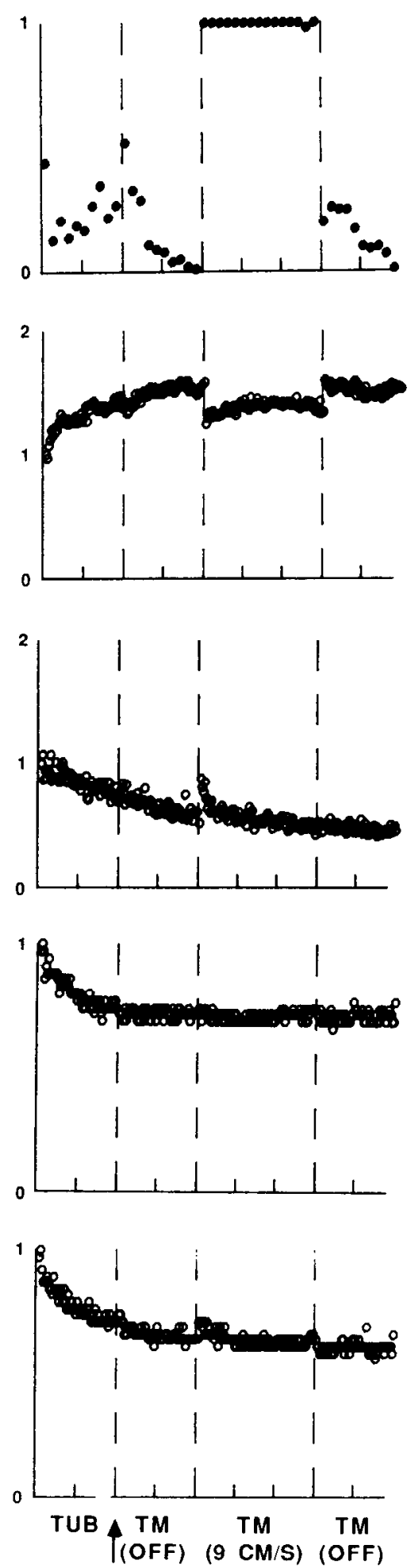

tions and for the TUB-to-TM transitions. Again, in each situation, the attenuation of EPSP amplitude was proportional not to the absolute value of the EPSP per se, but to the magnitude of EPSP growth just prior to the transition into either the RC $(F=8.156, p=0.0156$; Fig. $5 B)$ or the TM $(F=42.472, p=$ 0.0001 ; Fig. $5 C$ ). Similar experiments to those just described were carried out using stimulus intensities well below the threshold for producing a population spike. The results were very similar in terms of both the exploration related EPSP growth and the proportional attenuation associated with forced locomotion.

Evoked population spike. The population spike data for ex- 
A

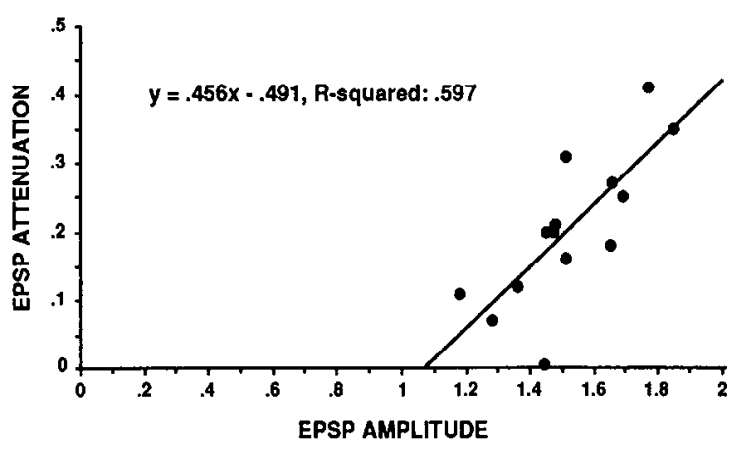

C

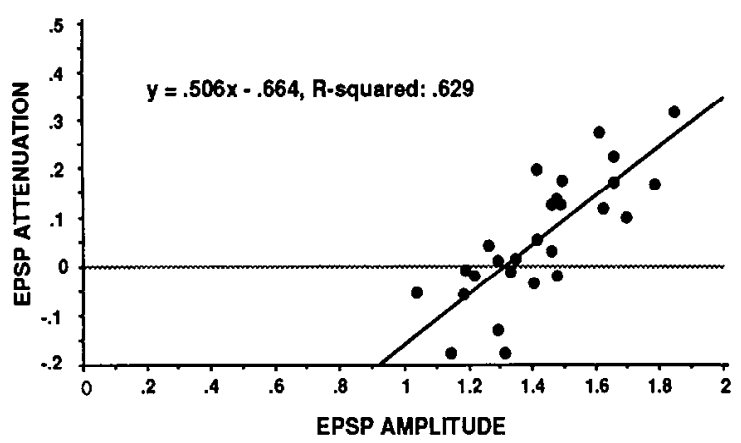

B

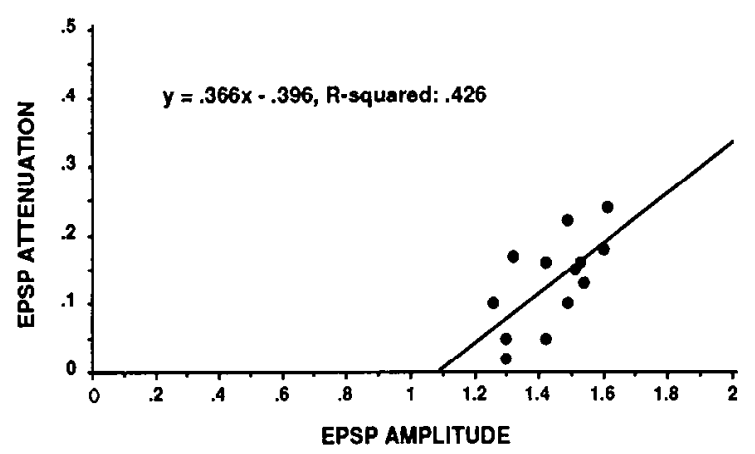

D

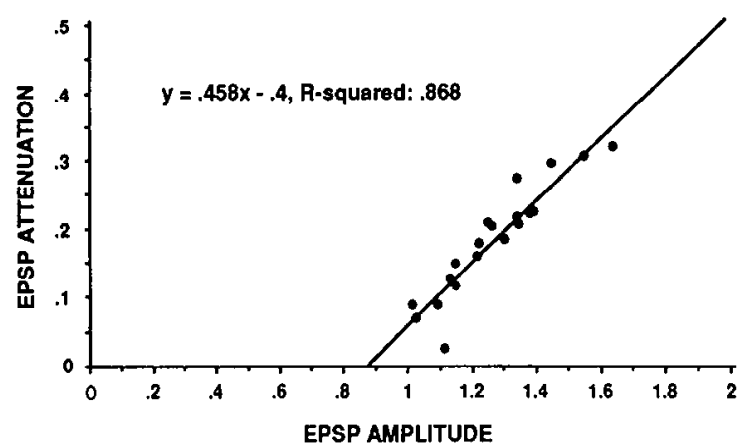

Figure 5. Relationship between EPSP amplitude and the magnitude of locomotion-related instantaneous EPSP reduction for 4 different environmental transitions. $A$, TM-treadmill transition (experiment 2a). $B$, TM-RC transition (experiment 2a). $C$, TUB-TM transition (experiment 2a). $D$, TM-treadmill transition (experiment 5). As indicated by the intercept of the regression lines near 1.0 on the EPSP amplitude axis, the transient reduction in EPSP amplitude was proportional to the growth of the EPSP above baseline immediately prior to the corresponding period of locomotion, and not to the absolute amplitude of the EPSP itself. Abbreviations as in Figure 4.

periment $2 \mathrm{a}$ are shown in the bottom 3 panels of Figure 4 . There was no significant change in the initial spike area values over the $6 \mathrm{~d}$ of this experiment $(F(5,10)=1.153, p>0.39)$, again indicating that any effects observed during a given day's recording had dissipated at least by the following day. Within sessions, spike area declined gradually over the first $20 \mathrm{~min}$ in the 2 session types ( $\min 1$ vs $\min 20 ; p<0.01$ ). A substantial, but transient, elevation in spike area occurred both when animals were transferred into the Plexiglas recording chamber (condition $\mathrm{A}$ ) and when the treadmill was turned on (condition $\mathrm{B}$ ).

Changes in spike onset and peak latency paralleled each other, dropping to near asymptotic levels over the first 10-15 min in both session types (min 1 vs $\min 20 ; p<0.01$ ). Final values for spike onset and peak latency were generally smaller for condition B than for condition A. This result is consistent with the greater final growth of the EPSP in the $\mathrm{B}$ condition. The relationship between EPSP growth and both spike onset latency and spike peak latency was further evaluated by plotting the fractional change in the latter measures as a function of the fractional EPSP growth over the initial $10 \mathrm{~min}$ following transfer into the TUB for each animal. In general, there was a statistically significant negative correlation between EPSP growth and spike onset latency whenever there was robust EPSP growth. The correlation between EPSP growth and spike area decline was less consistent.

Spike activation threshold. In order to estimate the relative extracellular synaptic current flow at the onset of granule cell population discharge (i.e., spike activation threshold), the EPSP amplitude at spike onset time was computed as described in Materials and Methods. These results are plotted in Figure $6 \mathrm{~A}$, along with the corresponding EPSP amplitudes (replotted from Fig. 4 in order to facilitate comparison). For statistical purposes, spike threshold values were compared at minutes $1,5,10,15$, and 20 for day 1 . As can be seen from the figure, there was no consistent change in EPSP amplitude at spike onset over this time, when EPSP growth was at a maximum $(F(4,20)=0.052$, $p>0.8$ ). Activation of the treadmill resulted in an abrupt decrease in activation threshold, which recovered immediately upon cessation of treadmill movement. Comparisons of threshold values for the 2 min immediately preceding and subsequent to activation of the treadmill indicated that this decrease was significant $(p<0.05)$. Introduction to the $\mathrm{RC}$ appeared to produce a similar, though transient, threshold decrease (Fig. $6 \mathrm{~A}$ ); however, this decrease was not statistically significant.

The failure of treadmill walking to produce significant EPSP growth suggests that the growth is not directly rclatcd to motor activity, but rather to the changes in sensory input that accompany exploratory behavior. A caveat to this conclusion, however, results from the possibility that EPSP growth had already reached maximum by the time treadmill walking was initiated. In order to explore this possibility, use was made of the fact that daily exposure to a particular environment results in a reduction of exploratory behavior and a concomitant reduction in EPSP growth. Exploration can be reinstated, however, by 
Figure 6, A, EPSP amplitude (top panels) and spike threshold (bottom panels) for experiment $2 \mathrm{a}$. RC condition (left column), and TM condition (right column). $B$. EPSP amplitude ( $t o p$ panels) and spike threshold (bottom panels) for day 1 (left column) and day 4 (right column) of experiment 5 . Notice that the spike threshold does not change during the phase of maximal EPSP growth, and that the reduction of the EPSP during vigorous locomotion is accompanied by a slight decrease in spike threshold. Abbreviations as in Figure 4.

\section{A}
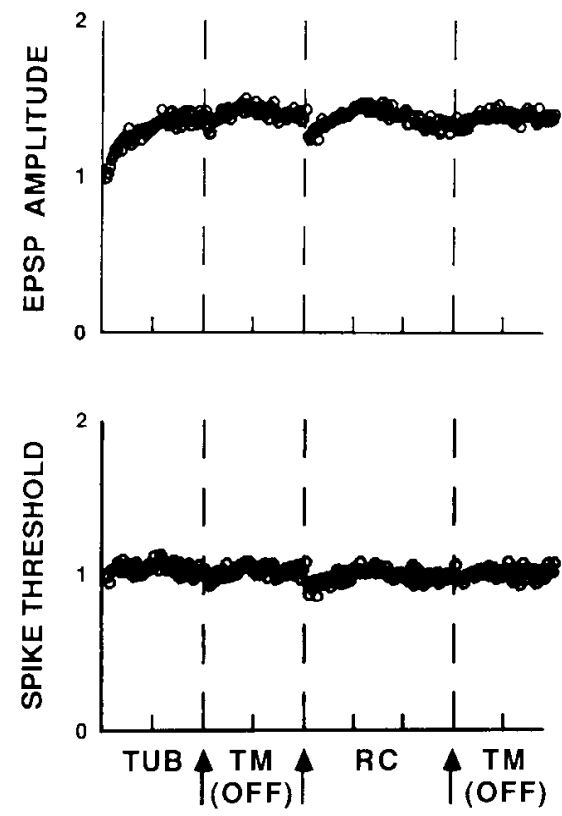

B
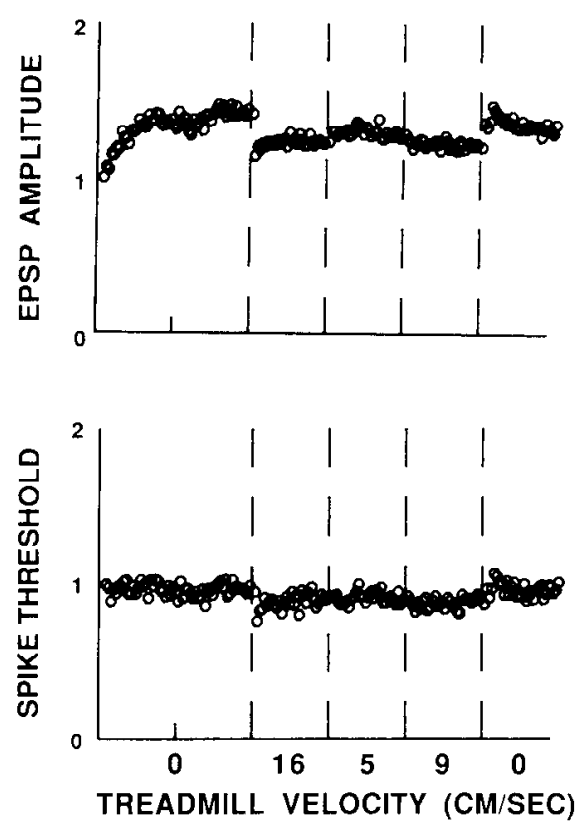
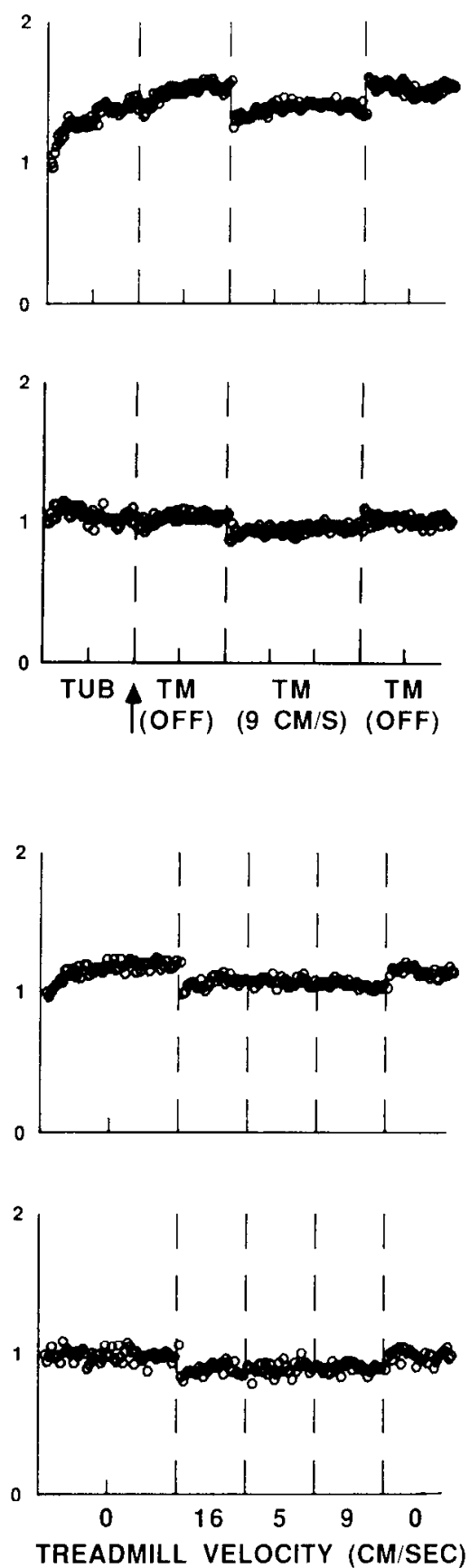

changing the environmental conditions in some way, for example, altering the ambient lighting. Thus, for experiment $2 \mathrm{~b}$, each of 10 rats was carried in a plastic tub to the recording room, connected to the recording apparatus, and returned to the tub. Following the initial $15 \mathrm{~min}$ of exploration in the plastic tub, cach rat was placed into the treadmill, moving at $9 \mathrm{~cm} / \mathrm{sec}$. $\Lambda \mathrm{fter}$ $2.5 \mathrm{~min}$ in the treadmill, the rat was returned to the plastic tub for an additional $12.5 \mathrm{~min}$. Five recording sessions were conducted using this procedure. The sixth recording session was conducted exactly like the first 5 , except that the animals were tested under red light conditions in order to facilitate exploration.

The results of this experiment are summarized in Table 1. Animals exhibited the greatest amount of exploration and EPSP growth on day 1, and a general decrease in both exploration and EPSP growth on days 2 through 5 . However, during the last testing session conducted under red light, both exploration and EPSP growth observed during the first $15 \mathrm{~min}$ attained levels similar to those observed in the first testing session (Table 1). Thus, the failure of the EPSP to grow during treadmill walking cannot be attributed to the presence of a growth "cciling" attained prior to the treadmill episode. Similar results are shown in Figure 9 (experiment 5).

\section{Experiment 3: role of hippocampal theta rhythm in evoked response alterations}

The preceding experiments demonstrated that neither motor activity nor the associated theta in the hippocampal EEG is 

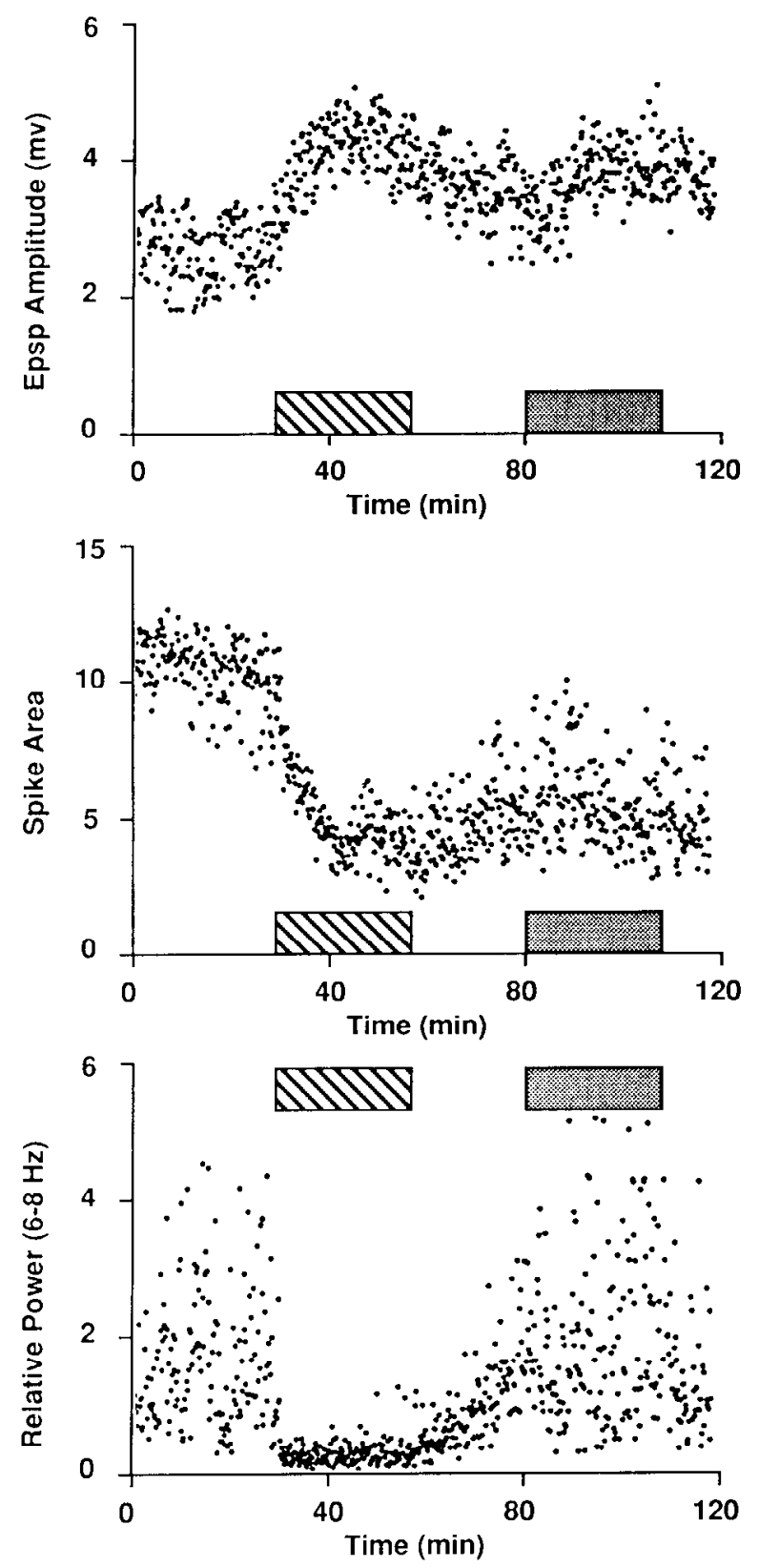
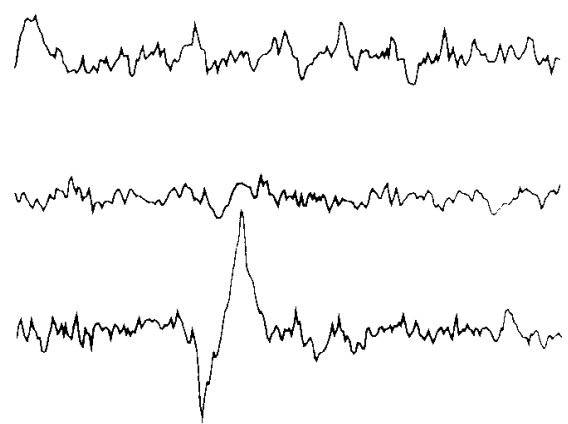

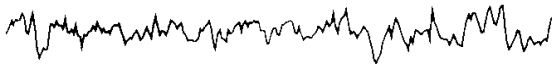
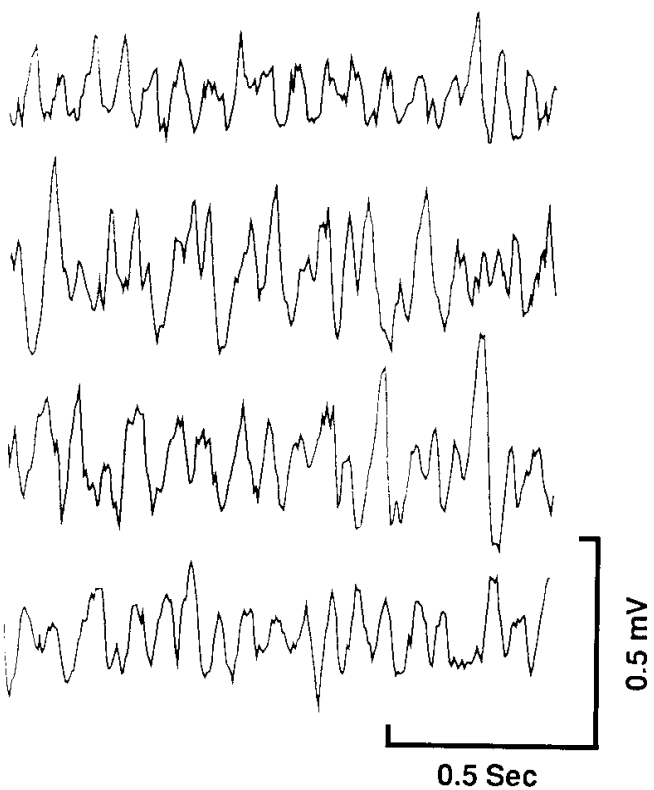

Figure 7. Experiment 3. The theta rhythm of the hippocampal EEG that normally accompanies exploratory activity or forced locomotion is unnecessary for the full expression of the exploration-associated evoked response alterations. In this experiment, the animal was placed overnight in the recording apparatus in its home cage, as in experiment 1 . After 30 -min baseline recording, $0.7 \mu$ l of a $2 \%$ solution of the local anesthetic tetracaine was injected into the medial scptal region via a chronically implanted cannula. As documented more fully by Mizumori et al. (1989a), this treatment was sufficient to abolish the hippocampal theta rhythm for 20 min or more. This is illustrated by representative EEG records (first 4 traces of right panel) and by the abrupt decline in relative EEG power in the 6-8- $\mathrm{Hz}$ band (lower left). Immediately following the injection, the animal was allowed $20 \mathrm{~min}$ free exploration of the platform on which the cage had rested (indicated by the cross-hatched bars) and was then returned to the home cage in the original location. A second 20 -min episode of exploration was allowed 40 min later (shaded bars), after the effects of the local anesthetic on the theta rhythm had worn off (see lower 4 EEG records, right panel) and the original response changes had recovered somewhat. This second period of exploration resulted in additional EPSP growth and spike depression. Abolition of the theta rhythm by tetracaine had no effect on the exploration-associated EPSP and population spike changes.

sufficient to produce the robust response alterations that accompany exploratory activity. The present experiment was conducted to determine whether hippocampal theta rhythm was a necessary prerequisite for these effects. The experiment was conducted using the same protocol as in experiment 1 , with the rat being left overnight in the recording apparatus. In the present experiment, however, $0.7 \mu$ l of $2 \%$ tetracaine was injected into the medial septum immediately prior to the exploratory session. As demonstrated by Mizumori et al. (1989a), this treatment is sufficient to abolish the hippocampal theta rhythm. Control sessions, in which no injections were made, were carried out in the same animal.

Injection of tetracaine into the medial septum resulted in a complete abolition of exploration-related hippocampal theta re- 
Table 1. Experiment 2B. Role of motor activity in evoked response alterations

\begin{tabular}{llll} 
& $\begin{array}{l}\text { Percent } \\
\text { exploration }\end{array}$ & \multicolumn{2}{c}{ Percent EPSP growth } \\
\cline { 3 - 4 } & Pretreadmill & Posttreadmill \\
\hline Session 1 & $39.9 \pm 2.3$ & $29.1 \pm 4.1$ & $29.5 \pm 3.4$ \\
Session 5 & $16.7 \pm 4.5^{a}$ & $13.8 \pm 5.5^{a}$ & $12.8 \pm 6.1$ \\
Session 6 (red light) & $36.9 \pm 2.4^{\circ}$ & $24.2 \pm 3.0^{h}$ & $25.4 \pm 5.2$
\end{tabular}

This table presents values (mean + SEM) of percent exploration, percent EPSP growth over baseline after $15 \mathrm{~min}$ of exploration (pretreadmill walking), and percent EPSP growth over baseline following a period of walking on the treadmill. Note the decrease in exploratory behavior between sessions 1 and 5 , and the corresponding reduction in pretreadmill EPSP growth. When exploration was facilitated by testing the animals under red light (session 6), both exploratory behavior and EPSP growth increased substantially (session 6 vs session $5, p<$ 0.01 ), indicating that the reduction in EPSP growth in session 5 was not due to changes in the potential (i.e., ceiling) for EPSP growth. Treadmill walking did not produce further increases in EPSP amplitude in any of the sessions (all comparisons, $p>0.2$ ). The results of a similar experiment are shown in Figure 9.

"Different from session $1(p<0.05)$.

${ }^{h}$ Different from session $5(p<0.01)$.

corded from the same electrode from which the evoked responses were obtained (see Fig. 7). Nevertheless, the period of exploration was accompanied by a substantial and persistent increase in the EPSP and a corresponding decrease in the evoked population spikc. The fractional changes in these components relative to baseline did not differ from control sessions.

\section{Experiment 4: evoked EPSP changes measured in the molecular layer}

Estimates of synaptic current flow recorded from the major current source in or below the stratum granulosum and from the site of synaptic activation in the stratum moleculare (the current sink) are generally assumed to parallel each other. However, recent observations indicate that, under certain conditions (i.e., different anesthetics and/or activation of subcortical afferents), the 2 may not always covary in a uniform manner (Dahl and Winson, 1985; Winson and Dahl, 1985; Pavlides et al., 1988). In this experiment, EPSP data were collected simultaneously from both sites in order to verify the general pattern of EPSP results obtained with stratum granulosum recordings. The electrodes in stratum moleculare also enabled an assessment of the presynaptic fiber response.

Two rats were surgically prepared with a stimulating electrode in the angular bundle, and a pair of recording electrodes mounted on a movable microdrive. The recording electrodes were mounted side-by-side on the microdrive such that the tips were separated (dorsoventrally) by about $400 \mu \mathrm{m}$. One day prior to the start of the formal recording sessions, the position of the electrode pair was adjusted until perforant path evoked responses recorded from the superficial electrode indicated that it was at the EPSP reversal point. The electrode pair was then withdrawn (dorsally) $180 \mu \mathrm{m}$ into the molecular layer. Thus, the evoked response was recorded simultaneously from 2 points near the principal sinks and sources of synaptic current, respectively.

For the recording sessions, each rat was carried in its plastic tub to the recording room, connected to the recording apparatus, and returned to the plastic tub. Perforant path stimuli were delivered at $0.1 \mathrm{~Hz}$, and evoked responses were sampled simultaneously from both recording electrodes at $20 \mathrm{kHz}$. Following an initial $10 \mathrm{~min}$ in the plastic tub (TUB), rats were tested for $10 \mathrm{~min}$ in the stationary treadmill (TM), followed by $15 \mathrm{~min}$ in the activated treadmill $(9 \mathrm{~cm} / \mathrm{sec})$ and a final $10 \mathrm{~min}$ in the stationary treadmill. Each rat received $4 \mathrm{~d}$ of testing.

Sample waveforms simultaneously recorded from both sites are shown in Figure $1 B$, and the results of the experiment are illustrated in Figure 8. The pattern and magnitude of EPSP fluctuations observed from electrodes at the 2 sites were essentially identical to one another. EPSPs recorded at both locations exhibited substantial $(>50 \%$ ) growth in amplitude over the initial $20 \mathrm{~min}$, and a reduction during the transition to the TM and when the treadmill was activated. One rat exhibited a prominent presynaptic fiber potential which was not substantially altered during any phase of these experiments. The fiber potential from the second animal was contaminated by stimulus artifact and was therefore not reliably measurable.

\section{Experiment 5: role of altered transmitter release probability in evoked response alterations as assessed by paired-shock depression ratios}

Two general classes of activity-dependent increases in synaptic efficacy have been recognized in the fascia dentata: (1) the relatively short-term increases in synaptic efficacy known as facilitation, augmentation, and potentiation (McNaughton, 1980, 1982), and (2) the longer-term increase in synaptic efficacy known as long-term synaptic enhancement (LTE), or long-term potentiation (LTP; Bliss and Gardncr-Medwin, 1973; Bliss and Lømo, 1973; Douglas and Goddard, 1975; McNaughton et al., 1978; Andersen et al., 1980). McNaughton $(1980,1982)$ has demonstrated that these short-term changes in synaptic efficacy can be observed at perforant path synapses, where they closely resemble similar changes at neuromuscular synapses (Magleby and Zengel, 1975, 1976a, b) and, as at neuromuscular junction, they result from a calcium-dependent increase in the fractional probability of transmitter release (the quantal parameter $p$ ). Conditions associated with large values of $p$ (such as augmentation and potentiation) act to increase transmitter depletion, and, hence, the magnitude of EPSP depression observed in the second of a closely spaced pair of perforant path responses. Conversely, conditions associated with small values of $p$, such as low extracellular calcium, act to decrease EPSP depression (McNaughton, 1980, 1982). In the following experiment, paired shocks were delivered to the perforant path in order to evaluate the possibility that alterations in the fractional probability of transmitter release could account for the EPSP growth associated with environmental exploration.

Stimulation and recording procedures were identical to those used in experiment $2 \mathrm{a}$ except that paired shocks ( $400 \mathrm{msec}$ ISI), rather than single shocks, were delivered to the perforant path at $0.1 \mathrm{~Hz}$. The 5 chronically prepared rats used in experiment 2a were transported to the recording room in their plastic tubs, connected to the recording apparatus, and then placed in the stationary treadmill. After $10 \mathrm{~min}$, the treadmill was turned on for $5 \mathrm{~min}$ at relatively high speed $(16 \mathrm{~cm} / \mathrm{sec})$, followed by 5 min at low speed $(5 \mathrm{~cm} / \mathrm{sec})$, and then $5 \mathrm{~min}$ at the intermediate speed used in experiment $2 \mathrm{a}(9 \mathrm{~cm} / \mathrm{sec})$. During the final $5 \mathrm{~min}$ the treadmill was stationary. Behavior was scored as described above. This experiment was carried out over 3 consecutive days. The procedure for the fourth day was similar to that used for days 1 through 3 , except that the treadmill was maintained at a constant speed $(9 \mathrm{~cm} / \mathrm{sec})$ for the entire 15 -min walk session, and, during the middle 5 min (minutes 16-20) of that session, the room lights were turned off. 
Behavior, EEG, and evoked EPSP. The results for days 1, 3, and 4 of this experiment are depicted in Figure 9. The results for day 2 were intermediate between those for days 1 and 3 and, for the sake of brevity, are not shown here. On day 1, considerable exploratory behavior and power in the EEG theta band was exhibited over the first $10 \mathrm{~min}$ of the session (in the stationary treadmill). This was accompanied by a large growth in the EPSP. Activation of the treadmill resulted in a precipitous momentary attenuation in EPSP amplitude. However, there was very little effect of treadmill velocity. When the treadmill was turned off, there was an immediate recovery of EPSP amplitude, followed by a gradual decline.

There was no significant variation in the initial EPSP values over the $4 \mathrm{~d}$ of the experiment $(F(3,12)=2.228, p>0.13)$. Exploratory behavior and theta power during the initial $10 \mathrm{~min}$ in the stationary treadmill declined over days (Fig. 9). Correspondingly, there was a significant reduction in EPSP growth during this period $(F(3,12)=9.848, p=0.0015)$. EPSP growth was greater on day 1 than on each of days 2,3 , and 4 (all $p<$ 0.05 ).

It is important to emphasize that in spite of the fact that the EPSP growth on day 4 was considerably less than the maximum value observed on day 1 , there was, nevertheless, virtually no growth of the EPSP during treadmill walking. Therefore, the failure of the EPSP to grow during treadmill walking cannot be attributed to a ceiling effect.

The reduction in the EPSP growth over the course of this experiment was accompanied by a decrease in the movementrelated EPSP attenuation during sessions on the moving treadmill. The relationship between EPSP and movement was assessed as described in experiment 2a. Again, the attenuation during walking showed a strong linear relationship to the prior growth of the $\operatorname{EPSP}(F(1,18)=118.04, p=0.0001$; see Fig. $5 D$ ) rather than to its absolute value.

EPSP amplitude at spike onset was also evaluated as described in experiment 2a, and the results are illustrated in Figure $6 B$. There was again no consistent change in spike onset threshold over the first $10 \mathrm{~min}$, the period of maximal EPSP growth (evaluated at minute 1,5 , and 10 of day $1 ; F(2,10)=0.018, p>$ 0.9). Walking on the treadmill resulted in a moderate, though significant, decrease in onset threshold at each treadmill speed (all $p<0.05$ ) but recovered immediately with cessation of treadmill movement.

Paired-shock effects. Paired-shocks (400 msec ISI) resulted in fractional EPSP changes which were similar in pattern (i.e., net EPSP depression) and in magnitude to those obtained from the medial perforant path in anesthetized and in vitro preparations (McNaughton, 1980). Figure 10 shows the average EPSP amplitude and the corresponding average fractional (within pair) EPSP change plotted as functions of time. There was no consistent change in fractional EPSP depression over the course of the first 10 min of the session, during the time of maximal EPSP growth (evaluated at minute 1,5 , and 10 of day $1 ; F(2,10)=$ $0.321, p>0.7)$. However, walking on the treadmill produced a moderate, though significant, reduction in fractional depression, which recovered during the final $5 \mathrm{~min}$ when the treadmill was stationary. Fractional EPSP values associated with treadmill walking at each speed were significantly lower than those during the minute just prior to activation of the treadmill and those during the 5-min period at the end of the session when the treadmill was stationary (all comparisons, $p<0.025$ ). A similar pattern of results (i.e., no significant change in fractional
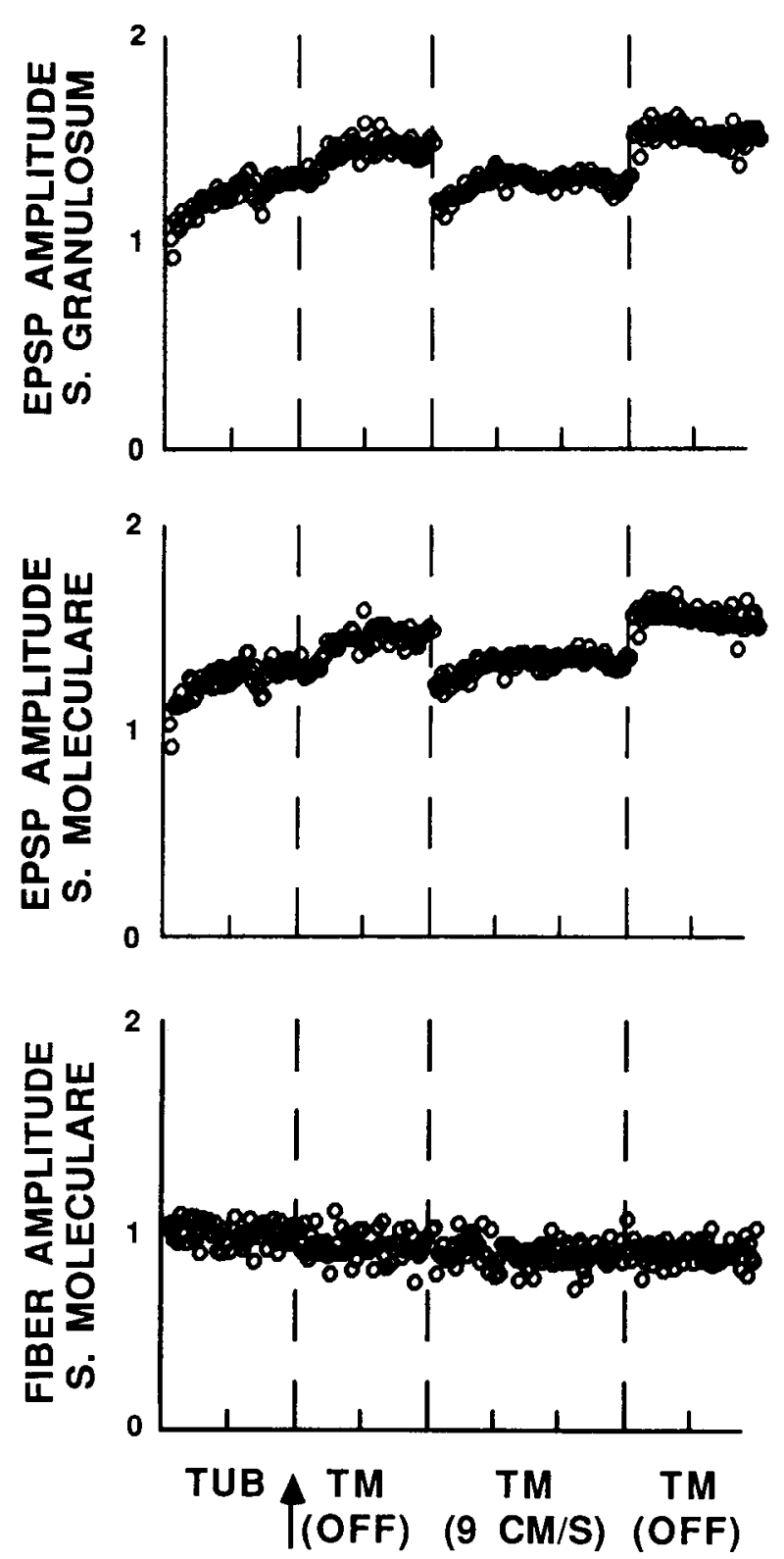

Figure 8. Experiment 4: Evoked EPSP data normalized relative to a baseline value of 1.0 , collected simultaneously from the stratum granulosum/hilus (top panel), and from the stratum moleculare (middle panel). The bottom panel shows presynaptic fiber potential data. These data indicate that the exploration-dependent EPSP growth is due neither to a significant shift in the distribution of current sources and sinks along the granule cell dendrites, nor to a change in the number of perforant path fibers activated by the stimulus. Abbreviations as in Figure 4.

depression during the period of maximal EPSP growth) was obtained using a between-shock interval of $30 \mathrm{msec}$.

Evoked spike. On day 1 the animals exhibited a reduction in spike area over the course of the first $10 \mathrm{~min}$ in the stationary treadmill, similar to that observed in previous experiments (Fig. 9). When the treadmill was turned on at high speed $(16 \mathrm{~cm} / \mathrm{sec})$, there was a transient elevation of spike area. By the cnd of the high-speed walk session, spike areas had returned to values approximately equal to those observed just prior to turning on the treadmill (minute 10). A reduction in treadmill speed to 5 $\mathrm{cm} / \mathrm{sec}$ also resulted in a transient increase in spike area. These 


\section{DAY 1}
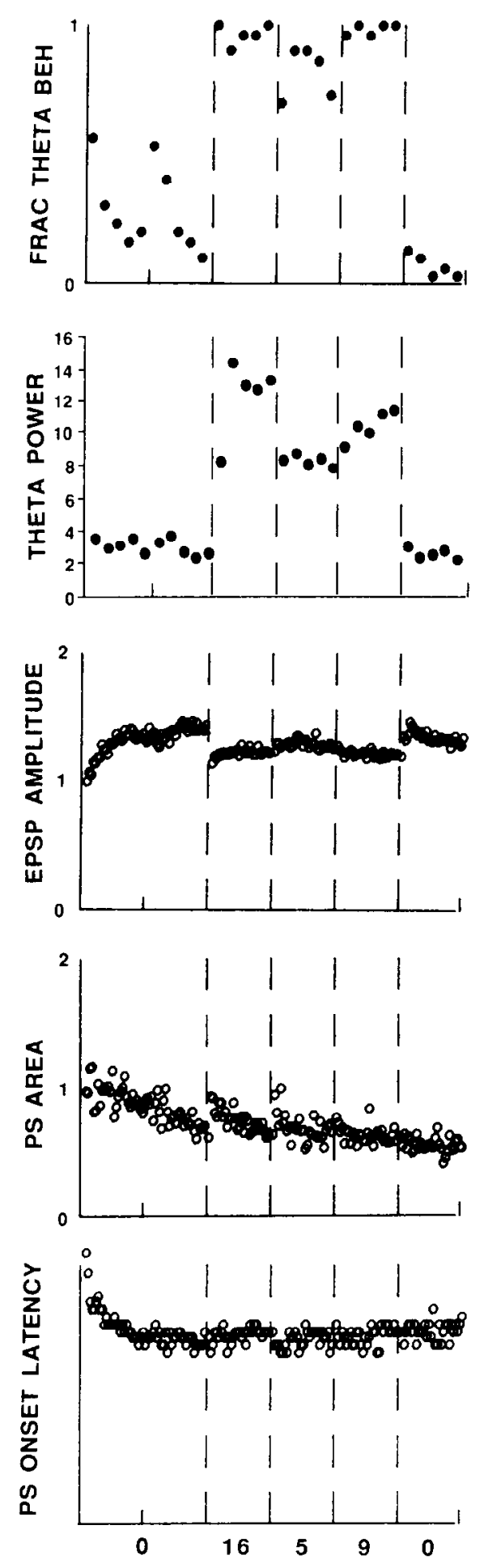

TREADMILL VELOCITY (CM/SEC)
DAY 3
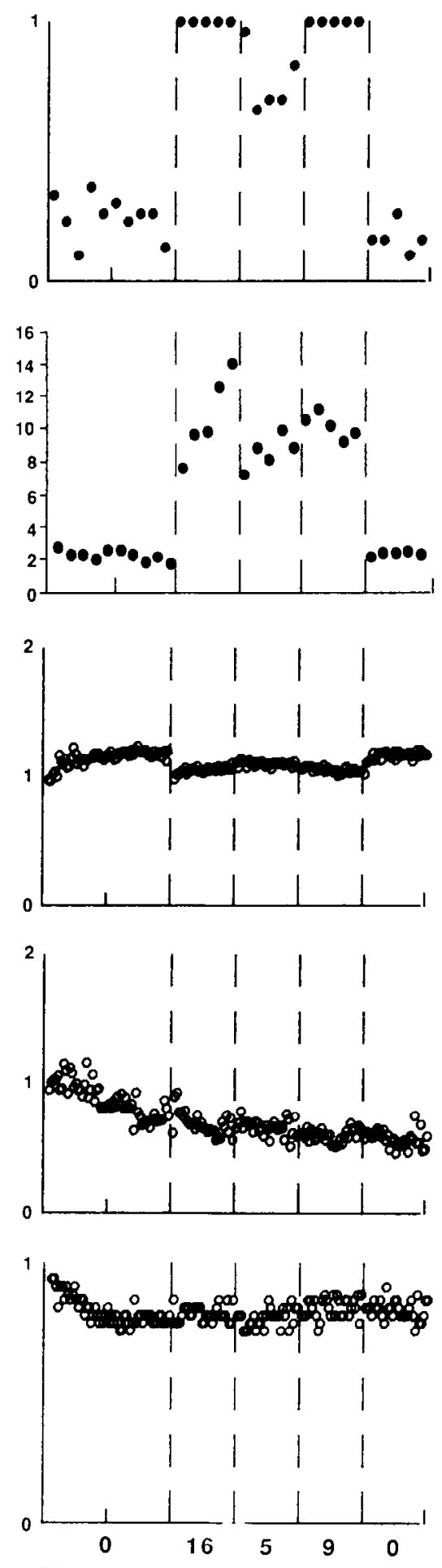

TREADMILL VELOCITY (CM/SEC)
DAY 4
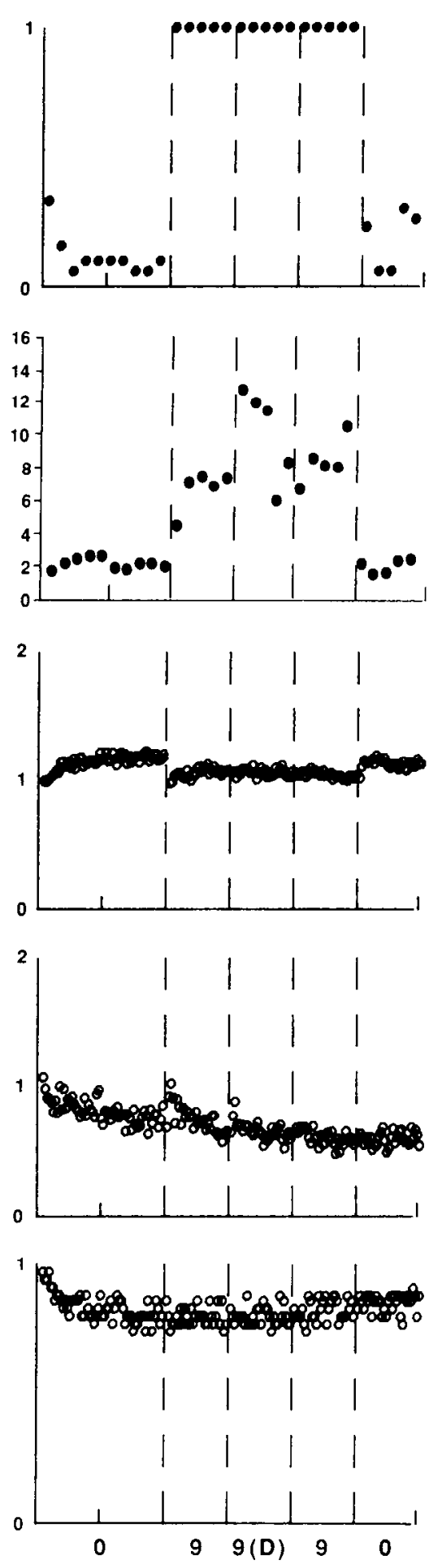

TREADMILL VELOCITY (CM/SEC)

Figure 9. Experiment 5: Treadmill velocity manipulations (see text). Average group means for the behavioral, EEG, and normalized evoked response parameters plotted as functions of time ( $5 \mathrm{~min} /$ division). The 3 columns show data from days 1,3 , and 4 , respectively. The second row shows the EEG spectral power between 6.6 and $7.4 \mathrm{~Hz}$ (averaged in 1 -min bins). It is important to note that, by day 4 , both exploratory behavior and theta activity during the initial $10 \mathrm{~min}$ had declined substantially. This decline was associated with a corresponding reduction in explorationdependent EPSP growth. Thus, EPSP growth at the end of the first 10 min on day 4 was nowhere near maximal. Nevertheless, the intense locomotion in the treadmill failed to induce further growth. $P S=$ population spike. 
transient increases were superimposed on a gradual decline that continued throughout the recording session.

There was no consistent change in the initial spike area values $(F(3,15)=1.683, p>0.23)$ or in the mean fractional spike decline during the first $10 \mathrm{~min}(F(3,15)=0.55, p>0.65)$ across the $4 \mathrm{~d}$ of this experiment.

Averages of spike area for the $30 \mathrm{sec}$ immediately preceding and subsequent to each transition were computed for each subject on each of days 1-3. A 2-factor (days and transition type within subject), repeated-measures ANOVA performed on these data revealed a significant effect of condition $(F(3,12)=6.743$, $p=0.0064)$, but no significant effect of day $(p<0.2)$ or day by condition interaction $(p>0.2)$. Post hoc comparisons indicated that the spike increases associated with the first transition $(0-$ $16 \mathrm{~cm} / \mathrm{sec}$ walk) were significantly greater than the subsequent transitions $(p<0.05)$. Averages of spike area for the $30 \mathrm{sec}$ immediately preceding and subsequent to turning off the room lights on day 4 revealed a small, transient increase in spike area associated with this manipulation (paired $t=2.182, p<0.05$, one tail).

Spike onset latency data for days 1,3, and 4 are illustrated in the bottom row of Figure 9. The relationship between EPSP growth, spike onset latency, and spike area was evaluated as in experiment $2 \mathrm{a}$. As observed in experiment $2 \mathrm{a}$, there was a significant negative correlation between spike onset latency and EPSP growth whenever EPSP growth was robust; the correlation between EPSP growth and spike area decline was somewhat less consistent (Table 2).

\section{Discussion}

The major effects of exploratory activity observed in the present study are consistent with those reported previously (Sharp et al.. 1986. 1989). Environmental transitions led to an increase in the incidence of exploratory behaviors and hippocampal theta activity, a substantial and persistent elevation in the size of the evoked field EPSP, and a reduction in the latencies to population spike onset and peak. There was a paradoxical decrease in spike magnitude. Of paramount interest is the fact that these changes in response parameters considerably outlasted the exploratory behaviors that induced them. In addition, a number of important new observations were made that aid in the interpretation of the possible mechanisms and functional significance of these effects.

First the EPSP growth was not associated with any change in the compound action potential of the presynaptic afferent fibers. Nor was there a change in the apparent threshold of the postsynaptic population spike. Thus, neither the EPSP growth nor the reduction in population spike onset latency can be at-
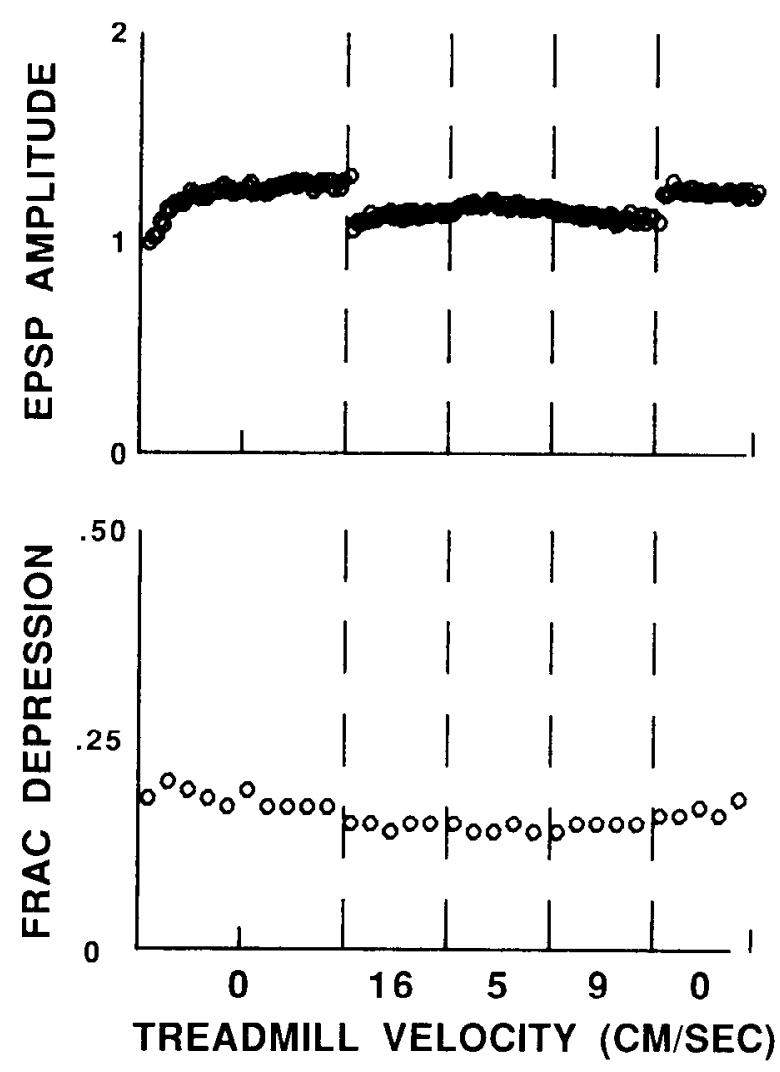

Figure 10. Experiment 5: Paired-shock interactions. Group means of normalized EPSP amplitude for the first of the paired responses (top) and fractional EPSP depression (bottom) plotted as functions of time for days $1-3$ of the experiment. The lack of an increase in the fractional depression parameter suggests that the growth of the EPSP is not due to an increased probability of transmitter release (see Discussion).

tributed to a change in either perforant path or granule cell excitability. EPSP growth was invariably accompanied by a general decline in the size of the evoked population spike. This decline was not increased by intense locomotor activity on the treadmill. It was, however, increased by additional bouts of exploration (e.g., Figs. 2 and 7). The decline in cvoked spike discharge was substantially more persistent than the clevation of the EPSP, suggesting that the 2 phenomena are not necessarily coupled.

Superimposed on the exploration-related spike decline was a transient elevation in spike amplitude. This occurred whenever the animal was exposed to a novel experience, such as transitions between environments, changes in room illumination, activa-

Table 2. Relationship between EPSP growth, spike onset latency, and spike area

\begin{tabular}{llll} 
Animal & $\begin{array}{l}\text { Percent EPSP } \\
\text { growth at min 10 }\end{array}$ & EPSP vs spike onset & EPSP vs spike area \\
\hline 755 & $64 \pm 4.6$ & $r=-0.503 ; p=0.0001$ & $r=-0.493 ; p=0.0001$ \\
756 & $55 \pm 1.4$ & $r=\cdots-0.267 ; p=0.039$ & $r=-0.144 ; p=0.2731$ \\
805 & $40 \pm 3.3$ & $r=\cdots 0.459 ; p=0.0002$ & $r=-0.372 ; p=0.0034$ \\
750 & $39 \pm 2.4$ & $r-\cdots 0.386 ; p-0.0023$ & $r=-0.180 ; p=0.1677$ \\
745 & $12 \pm 3.2$ & $r=-0.086 ; p=0.5117$ & $r=-0.229 ; p=0.0786$
\end{tabular}

Tata are from the first day of experiment 5 . The columns depict, from left to right, the animal identification, percent EPSP growth ( \pm SEM) at minute 10 , regression statistics for EPSP amplitude vs spike onset, and for EPSP amplitude is spike area (sce text for details). 


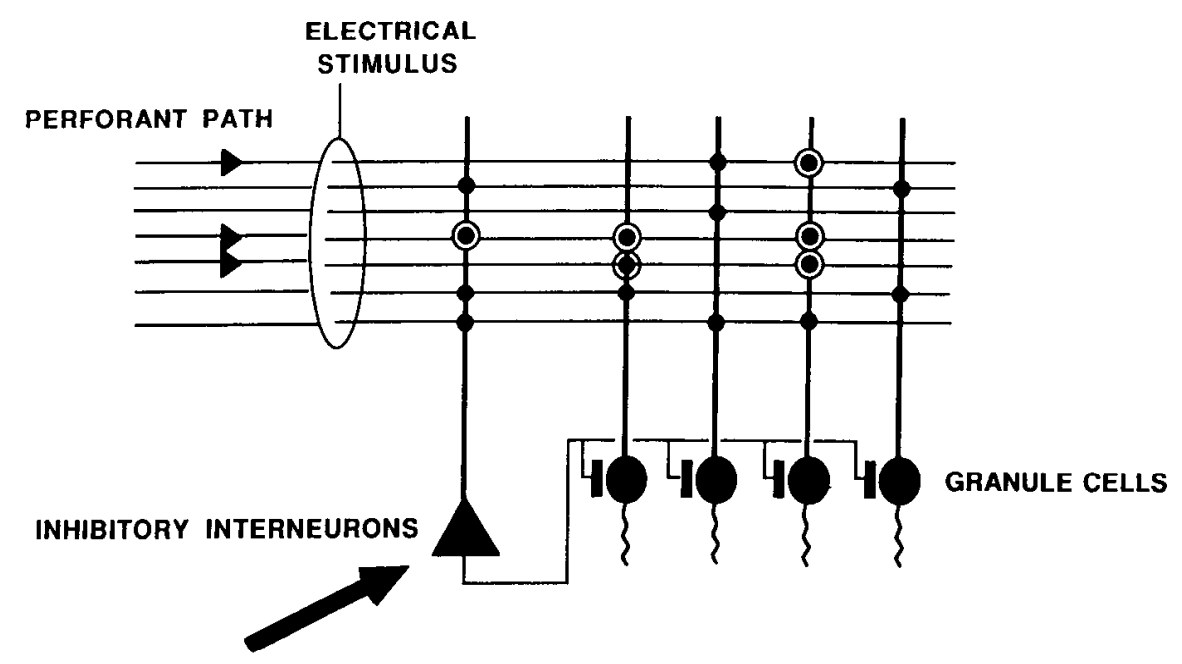

POSSIBLE SUBCORTICAL MODULATION

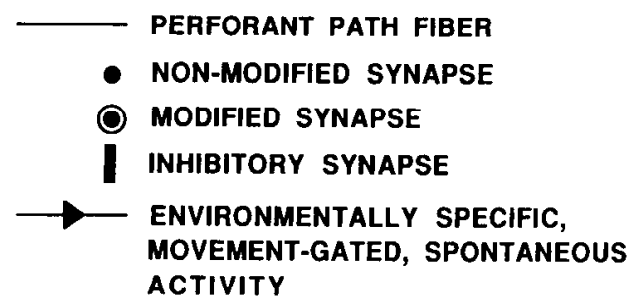

Figure 11. Schematic summary of the hypothesis proposed to account for the sequence of response changes that accompany environmental exploration. We propose that exploration in a particular environment may rcsult in the increased firing of a small, specific subset of perforant path fibers, those which are driven by the particular sensory features present in that environment. This elevation in firing rate could result in the activation of some form of synaptic strengthening in the terminals of the active fibers, which would be manifested as an increase in evoked field EPSPs. However, during intense locomotion, the increase in granule cell depolarization associated with tonic activity of the strengthened synapses would reduce the evoked synaptic current flow in response to perforant path stimulation. Under the assumptions that (1) the modified synapses are the same ones that are spontaneously active during walking in a given environment, and (2) that nonmodified synapses are relatively weak, the reduction in observed EPSP would be in proportion to the total number of modified synapses activated by the stimulating electrode. Hence, the walking induced momentary EPSP reduction would be proportional to the net growth of the EPSP rather than to its absolute amplitude. To account for the apparent paradox that exploration causes a simultaneous reduction in spike amplitude and spike onset latency, we assume that synaptic strengthening also occurs at some perforant path synapses onto inhibitory interneurons. This would produce a general increase in evoked feedforward inhibition, which would result in suppression of firing of the longer latency units contributing to the population spike, hence shifting the apparent peak to the left. Alternatively, the same general effect could result from the modulation of interneuron excitability by afferents from other neural structures.

tion of the treadmill, or simply changes in its speed. Thus, this component of evoked spike alteration might be related to novelty.

Second, simultaneous recordings from the stratum moleculare and the stratum granulosum/hilus provide strong evidence that the EPSP growth observed in these experiments did not result simply from a change in the cable properties of granule cell dendrites. An increase in dendritic membrane resistance, for example, might increase the transfer of synaptic charge to the granule cell somata and hence act to produce greater source current flow in this region. Such an alteration would be consistent with the reduction in spike onset latency that accompanies EPSP growth. However, the magnitude and pattern of EPSP growth recorded in the stratum granulosum/hilus and stratum moleculare were essentially identical. Thus, EPSP growth cannot be accounted for by a redistribution of current sinks and sources. In addition, this observation confirms that the EPSP changes are generated in the fascia dentata and do not result from volume conduction from other regions.

Third, as noted by Sharp et al. (1989), during periods of intense locomotor activity such as transitions between environments or, as in the present experiments, treadmill walking, there was a substantial, but transient, decline in the field EPSP amplitude. The proportional reduction of EPSP amplitude during treadmill walking was independent of stimulus intensity over a broad range. The EPSP reduction recovered immediately upon cessation of the treadmill activity. Sharp et al. (1989) reported that this sort of attenuation did not occur in the absence of prior EPSP growth. In the present studies, we found that the attenuation was strictly proportional to the magnitude of the EPSP growth just prior to locomotor activity, rather than to the absolute amplitude of the EPSP itself. This observation indicates that it is only the modified component of the population EPSP that is subject to the locomotion-induced transient suppression. A possible implication of this is that there are 2 separate groups of synapses contributing to the modified and nonmodified components, respectively.

Intense locomotor activity was also accompanied by a transient decline in the apparent spike threshold. Such a decline in field EPSP and apparent spike threshold would be expected, for example, if the granule cells were subject to a tonic depolarizing influence. Apart from this threshold change, there were no consistent changes in population spike parameters resulting from stationary locomotor activity in the treadmill. 
Particularly important was the finding that treadmill activity did not lead to the same sort of persistent alterations that were induced by comparable periods of exploratory activity. Thus, intense locomotion on the treadmill and the accompanying robust theta rhythm in the EEG failed to induce significant EPSP growth, even though levels of pretreadmill EPSP growth (and exploratory behavior) were quite low (experiment 5, Fig. 9). That the general reduction in initial EPSP growth was related to the reduced exploration, and not to a change in the potential for EPSP growth, is supported by the results from experiment $2 b$ (see Table 1). In that experiment, animals exhibited a general reduction in both exploratory behavior and EPSP growth over the course of 5 testing sessions, but showed substantial increases in EPSP growth during a sixth session in which exploration was facilitated by testing the animals under a red light. Taken together, the data strongly suggest that neither locomotion per se nor the increased hippocampal theta rhythm are sufficient to account for the persistent response alterations.

As a further control, experiments in which baseline conditions were recorded prior to environmental transitions demonstrated that the EPSP growth was not simply a recovery from the depression that is associated with exploration-related or other locomotion. Moreover, as the home cage in which the baseline recordings were carried out was transparent and uncovered, general sensory adaptation cannot account for the observed response changes.

Fourth, injection of the local anesthetic tetracaine into the region of the medial septal nucleus resulted in complete abolition of the hippocampal theta rhythm during exploration. Nevertheless, this failed to affect the persistent alterations in the evoked response. Thus, hippocampal theta rhythm is not necessary for these changes, which indeed appear completely unrelated to the hippocampal EEG state. Although other interpretations are possible, the dissociation of the observed response changes from both the locomotor and EEG correlates of exploration leads strongly to the conclusion that these changes must be due to the variation in sensory input that accompanies exploration.

Finally, growth of the EPSP was not accompanied by any change in the fractional EPSP depression in response to paired stimulation of the perforant path. Previous studies have shown that conditions which alter transmitter relcasc probability, such as altered extracellular $\mathrm{Ca}^{2+}$ or the induction of short-term facilitation, augmentation, and potentiation, result in substantial changes in the relative sizes of EPSP responses to paired shocks (McNaughton, 1980, 1982). Although additional factors may also contribute to the observed paired shock effects, the fact that no changes were observed during the period of maximal EPSP growth in the present study indicates that the mechanism of the exploration-related growth does not involve these particular presynaptic processes. It is of interest that high-frequency-stimulation-induced LTE/LTP is also not accompanied by such changes (McNaughton, 1982). This leaves open the possibility that exploration-induced EPSP growth and LTE may share elements of a common mechanism. However, any such relationship remains to be demonstrated, perhaps through pharmacological blockade of the NMDA receptor complex.

Sharp ct al. (1989) considered several possible classes of explanation for the main effects of exploration on the field EPSP and population spike, including (1) increased perforant path synaptic efficacy, (2) increased extracellular current flow due to hyperpolarization of the granule cell population, or (3) a com- bination of these 2 processes occurring on different elements of the population.

In the present study, the reduction in spike onset latency during the period of maximal EPSP growth argues against hyperpolarization of the granule cells. Hyperpolarization should have increased spike latency, as, for example, occurs during recovery from paired pulse inhibition. Moreover, the stability of the apparent spike threshold is also inconsistent with the hyperpolarization hypothesis. Hyperpolarization of a subset of the granule cells should have produced an increase in the net extracellular current flow at spike threshold, unless it was exactly matched by an equivalent reduction in current flow (i.e., a depolarization) from other granule cells, in which case there should have been no net EPSP growth. The sensitivity of the measurements on the field EPSP to detect such changes in polarization state is supported by the observation that application of L-glutamate to granule cell dendrites in vitro decreases both the field EPSP and the apparent spike threshold (Green and Rao, unpublished observations). Taken together, the foregoing considerations indicate that the persistent EPSP growth that accompanies exploration reflects a true increase in evoked synaptic efficacy in granule cells.

\section{General hypothesis}

The pattern of results observed in these experiments is sufficiently complex to preclude any simple, unitary explanation. The following hypothesis, however, accounts at least in part for a number of the main effects (Fig. 11) and is proposed primarily for the purpose of generating testable predictions to guide future experiments. We propose that exploratory movement in a given environment results in the increased firing of a small, specific set of perforant path afferents which are driven by the particular sensory features present in that environment. As most hippocampal and entorhinal cortical neurons are strongly modulated by locomotion (Ranck, 1973; Fox and Ranck, 1975; Rose et al., 1983; Quirk et al., 1987), we assume that the selected afferents are strongly activated only during movement. This elevation in firing rate, we suppose, results in the activation of some relatively persistent form of synaptic strengthening in at least some of the terminals of the fibers concerned.

If the elevation in synaptic efficacy at the selected synapses were rather substantial relative to the "ground state," the increase in granule cell depolarization associated with tonic activity of those synapses during locomotion would reduce the evoked synaptic current flow in response to perforant path stimulation, in much the same way that application of L-glutamate to hippocampal cells in vitro reduces the evoked field EPSP (Collingridge et al., 1983; Green and Rao, unpublished observations). This would explain the reduced EPSP and spike threshold observed during walking and could account for the fact that the abrupt attenuation of EPSP values during intense locomotion is proportional to its previous growth rather than to its absolute magnitude.

The persistent decline in spike amplitude resulting from exploration and the transient spike increase that accompanies novelty remain to be accounted for. At least 3 different factors might contribute to these effects. One is that perforant path synapses onto inhibitory interncurons also increase in strength (Buzsaki and Eidelberg, 1982; Kairiss et al., 1987; Taube and Schwartzkroin, 1987), and that this increase is more persistent than that which occurs on granule cells. The resulting increase in feedforward inhibition of granule cells could account for the reduced 
spike. A second possibility is that of persistent changes at the inhibitory synapse itself. However, this possibility seems unlikely since it would be expected to have led to a general elevation of spike threshold, which was not observed. Finally, the general decline in spike amplitude and the transient increase associated with novelty might both result from changes in the excitability of inhibitory neurons due to the influence of some secondary (possibly subcortical) source. A number of such subcortical afferents can modulate granule cell excitability without any apparent effects on the EPSP; these include the medial septum (e.g., Alvarez-Leefmans and Gardner-Medwin, 1975; Fantie and Goddard, 1982; Robinson and Racine, 1986), median raphe (e.g., Winson, 1980), locus coeruleus (Winson and Dahl, 1985), nucleus parafascicularis (Dahl and Winson, 1986), and supramammillary nucleus (Winson, 1981; Mizumori et al., 1989b). The involvement of locus coeruleus and/or the raphe nuclei seems particularly plausible given the propensity of some cells in these areas to be activated by novel stimuli (e.g., AstonJones and Bloom, 1981) and under conditions of behavioral arousal (e.g., Trulson and Jacobs, 1979).

We cannot yet rule out the possibility that the cellular changes underlying the phenomena described are distributed throughout the population in a nonspecific manner in which there is no mapping between the particular neuronal elements affected and the particular environment explored. Nevertheless, the increased synaptic current and decrease in evoked granule cell discharge resulting from environmental exploration suggest the possibility that exploration may result in a substantial increase in the specificity of granule cell output associated with a particular environment. The most consistent explanatory hypothesis we are able to suggest is based on the assumption of such selectivity. One function of such a change might be the formation of an efficient representation of the local environment involving relatively few granule cells.

\section{References}

Alvarez-Leefmans FJ, Gardner-Medwin AR (1975) Influences of the septum on the hippocampal dentate area. J Physiol 249:14P-16P.

Andersen P, Sundberg SH, Sveen O, Swann JN, Wigström H (1980) Possible mechanisms for long-lasting potentiation of synaptic transmission in hippocampal slices in guinea-pigs. J Physiol 302:463-482.

Aston-Jones G, Bloom FE (1981) Norepinephrine-containing locus coeruleus neurons in behaving rats exhibit pronounced responses to non-noxious environmental stimuli. J Neurosci 1:887-900.

Barnes CA (1979) Memory deficits associated with senescence: a neurophysiological and behavioral study in the rat. J Comp Physiol Psychol 93:74-104.

Bliss TVP, Gardner-Medwin AR (1973) Long-lasting potentiation of synaptic transmission in the dentate area of the unanaesthetized rabbit following stimulation of the perforant path. J Physiol 232:357-374.

Bliss TVP, Lømo T (1973) Long-lasting potentiation of synaptic transmission in the dentate area of the anaesthetized rabbit following stimulation of the perforant path. J Physiol 232:331-356.

Buzsaki G, Eidelberg E (1982) Direct afferent excitation and long-term potentiation of hippocampal interneurons. J Neurophysiol 48:597607.

Collingridge GL, Kehl SJ, McLennan H (1983) Excitatory amino acids in synaptic transmission in the Schaffer collateral-commissural pathway of the rat hippocampus. J Physiol 334:33-46.

Dahl D, Winson J (1985) Action of norepinephrine in the dentate gyrus. I. Stimulation of locus coeruleus. Exp Brain Res 59:491-496.

Dahl D, Winson J (1986) Influence of neurons of the parafascicular region on neuronal transmission from perforant pathway through dentate gyrus. Brain Res 377:391-396.

Douglas RM, Goddard GV (1975) Long-term potentiation of the perforant path-granule cell synapse in the rat hippocampus. Brain Res $86: 205-215$.
Fantie BD, Goddard GV (1982) Septal modulation of the population spike in the fascia dentata produced by perforant path stimulation in the rat. Brain Res 252:227-237.

Fox SE, Ranck JB Jr (1975) Localization and anatomical identification of theta and complex-spike cells in dorsal hippocampal formation of rats. Exp Neurol 49:299-313.

Jarrard LE (1983) Selective hippocampal lesions and behavior: effects of kainic acid lesions on performance of place and cue tasks. Behav Neurosci 97:873-889.

Kairiss EW, Abraham WC, Bilkey DK, Goddard GV (1987) Field potential evidence for long-term potentiation of feed-forward inhibition in the rat dentate gyrus. Brain Res 401:87-94.

Magleby KL, Zengel JE (1975) A quantitative description of tetanic and post-tetanic potentiation of transmitter release at the frog neuromuscular junction. J Physiol 245:183-208.

Magleby KL, Zengel JE (1976a) Augmentation: a process that acts to increase transmitter release at the frog neuromuscular junction. $\mathbf{J}$ Physiol 257:449-470.

Magleby KL, Zengel JE (1976b) Long-term changes in augmentation, potentiation, and depression of transmitter release as a function of repeated synaptic activity at the frog neuromuscular junction. J Physiol 257:471-494.

McNaughton BL (1980) Evidence for two physiologically distinct perforant pathways to the fascia dentata. Brain Res 199:1-19.

McNaughton BL (1982) Long-term synaptic enhancement and shortterm potentiation in rat fascia dentata act through different mechanisms. J Physiol 324:249-262.

McNaughton BL, Douglas RM, Goddard GV (1978) Synaptic enhancement in fascia dentata: cooperativity among coactive afferents. Brain Res 157:277-293.

McNaughton BL, Barnes CA, Rao G, Baldwin J, Rasmussen M (1986) Long-term enhancement of hippocampal synaptic transmission and the acquisition of spatial information. J Neurosci 6:565-571.

Mizumori SJY, Barnes CA, McNaughton BL (1989a) Reversible inactivation of the medial septum: selective effects on the spontaneous unit activity of different hippocampal cell types. Brain Res 500:99106.

Mizumori SJY, McNaughton BL, Barnes CA (1989b) A comparison of septal and supramammillary influences on hippocampal unit activity and field potentials. J Neurophysiol 61:1-17.

Morris RGM, Garrud P, Rawlins JNP, O'Keefe J (1982) Place navigation impaired in rats with hippocampal lesions. Nature 297:681683.

O'Keefe J, Nadel L (1978) The hippocampus as a cognitive map. Oxford: Clarendon.

Olton DS, Walker JA, Gage FH (1978) Hippocampal connections and spatial discrimination. Brain Res 139:295-308.

Quirk GJ, Muller RU, Kubie JL, Ranck JB Jr (1987) Hippocampal and entorhinal place cells: what happened when the lights went out. Soc Neurosci Abstr 13:1331.

Pavlides C, Greenstein YJ, Winson J (1988) Comparative extracellular current flow at dendrites and soma of dentate granule cells during long-term potentiation. Neurosci Lett 92:177-181.

Ranck JB Jr (1973) Studies on single neurons in dorsal hippocampal formation and septum in unrestrained rats. I. Behavioral correlates and firing repertoires. Exp Neurol 41:461-555.

Robinson GB, Racine RJ (1986) Interactions between septal and entorhinal inputs to the dentate gyrus: facilitation effects. Brain Res 379: 63-67.

Rose G, Diamond D, Lynch GS (1983) Dentate granule cells have the behavioral characteristics of theta neurons. Brain Res 266:29-37.

Sharp PE, McNaughton BL, Barnes CA (1985) Enhancement of hippocampal field potentials in rats exposed to a novel, complex environment. Brain Res 339:361-365.

Sharp PE, McNaughton BL, Barnes CA (1986) Intermediate-term effects of environmental input on the perforant path evoked population EPSP of dentate gyrus granule cells. Soc Neurosci Abstr 12:520.

Sharp PE, McNaughton BL, Barnes CA (1989) Exploration dependent modulation of evoked responses in fascia dentata: fundamental observations and time course. Psychobiology 17:257-269.

Sutherland RJ, Wishaw IQ, Kolb B (1983) A behavioral analysis of spatial localization following electrolytic, kainate- or colchicine-induced damage to the hippocampal formation in the rat. Behav Brain Res 7:133-153.

Taube JS, Schwartzkroin PA (1987) Intracellular recording from hip- 
pocampal CAl interneurons before and after development of longterm potentiation. Brain Res 419:32-38.

Trulson ME, Jacobs BL (1979) Raphe unit activity in freely moving cats: correlation with level of behavioral arousal. Brain Res 163:135150.

Vanderwolf $\mathrm{CH}$ (1969) Hippocampal electrical activity and voluntary movement in the rat. Electroenceph Clin Neurophysiol 26:407-418.

Winson J (1974) Patterns of hippocampal theta rhythm in the freely moving rat. Electroenceph Clin Neurophysiol 36:291-301.

Winson J (1980) Influence of raphe nuclei on neuronal transmission from perforant path through dentate gyrus. J Neurophysiol 44:937950.
Winson J (1981) Reticular formation influence on neuronal transmission from perforant pathway through dentate gyrus. Brain Res 225:37-49.

Winson J, Abzug C (1977) Gating of neuronal transmission in the hippocampus: efficacy of transmission varies with behavioral state. Science 196:1223-1225.

Winson J, Abzug C (1978) Neuronal transmission through hippocampal pathways dependent on behavior. J Neurophysiol 41:716-732.

Winson J, Dahl D (1985) Action of norepinephrine in the dentate gyrus. II. Iontophoretic studies. Exp Brain Res 59:497-506. 